\title{
Platelet's Fatty Acids and Differential Diagnosis of Major Depression and Bipolar Disorder through the Use of an Unsupervised Competitive-Learning Network Algorithm (SOM)
}

\author{
Serena Benedetti ${ }^{1}$, Sabina Bucciarelli2 ${ }^{2}$, Franco Canestrari' ${ }^{1}$, Simona Catalani', \\ Silvia Mandolini' ${ }^{3}$, Valerio Marconi' ${ }^{1}$, Anna Rita Mastrogiacomo', Raffaella Silvestri ${ }^{3}$, \\ Maria Chiara Tagliamonte1, Raimondo Venanzini' ${ }^{3}$, Giuseppe Caramia ${ }^{2,4}$, Fabio Gabrielli2, \\ Lucio Tonello ${ }^{2 *}$, Massimo Cocchi ${ }^{*}$ \\ ${ }^{1}$ Department of Biomolecular Science, University of Urbino "Carlo Bo", Urbino, Italy \\ "Paolo Sotgiu" Institute for Research in Quantum Psychiatry \& Cardiology, LU de S University, Lugano, \\ Switzerland \\ ${ }^{3}$ Department of Mental Health, Area Vasta 1, Fano, Italy \\ ${ }^{4}$ Emeritus Professor Children Hospital, Ancona, Italy \\ Email: "luciotonello@gmail.com, ${ }^{*}$ massimo.cocchi@unibo.it
}

Received 25 March 2014; revised 1 May 2014; accepted 8 May 2014

Copyright (C) 2014 by authors and Scientific Research Publishing Inc.

This work is licensed under the Creative Commons Attribution International License (CC BY).

http://creativecommons.org/licenses/by/4.0/

(c) (i) Open Access

\section{Abstract}

Aim of the study: The main purpose of this work was to verify, through the study of three fatty acids of platelets (namely: Palmitic Acid-PA, Linoleic Acid-LA, Arachidonic Acid-AA) the ability to distinguish adult subjects with Major Depression (MD) from those with Bipolar Disorder (BD), using an artificial neural network (Self Organizing Map-SOM) and an indirect index of the viscosity of the membrane (B2), in agreement with previous results; secondly, the ability to understand any similarities between children and adults in the molecular characterization of mood disorders, both in general and in relation to a subset of individuals with suicidal ideation, indicated by the survey instruments used (SOM). Design: The study design, in order to achieve the objectives, has forecast the recruitment of three groups of subjects without regard to sex, age, food intake patterns, or pharmacological therapies: 1) Controls; 2) Subjects with Major Depression; 3) Subjects with Bipolar Disorder. They were provided for the following investigations: 1) Platelet Fatty Acids analysis; 2) Plasma and platelet serotonin levels; 3) Oxidative stress and inflammation markers.

\footnotetext{
${ }^{*}$ Corresponding authors.
}

How to cite this paper: Benedetti, S. et al. (2014). Platelet's Fatty Acids and Differential Diagnosis of Major Depression and Bipolar Disorder through the Use of an Unsupervised Competitive-Learning Network Algorithm (SOM). Open Journal of Depression, 3, 52-73. http://dx.doi.org/10.4236/ojd.2014.32011 
Moreover, the data of the fatty acids of platelets, previously obtained by a group of children were used, for comparison, in the SOM, with adults surveyed. Subjects participating in the study: All participants, volunteer, were recruited in the judgment of psychiatrists as they presented themselves to the office visit of the "Dipartimento di Salute Mentale", ASUR 4, Fano, Italy. All subjects were submitted to a semi-structured interview based on DSM-IV-TR criteria. 21-item Hamilton Depression Rating Scale (HDRS), Hamilton Anxiety Rating Scale (HARS), Clinical Global Impressions Scale (CGI), Symptom Checklist-90 (SCL-90), and Hypomania/Mania Symptom Checklist (HCL-32) were also applied to each patient. Adult subjects who did not give consent, patients with a diagnosis other than an affective disorder and subjects in their first clinical episode were excluded. The study was double blind. Outcomes: The results obtained have confirmed the main objective of the study. It was possible to obtain, in fact, the recognition of individuals with Major Depression and Bipolar Disorder, using the SOM and the index B2. Value of the study: The methodology used in this study may be of utility, such as quantitative diagnostic support to the psychiatrist, in order to reduce the high error that occurs in the first diagnosis, with regard to mood disorders. Limitations of this study: The limitations of this study are mainly related to the number of controls. They should have been more numerous such as the number of suicidal ideations. About the comparison with children, the main limitation seems to be the lack of psychiatric diagnosis of children, so that any results just assume the value of hypotheses. Future Research: Because of the strong classificatory properties of the SOM, it would require a RCT in a larger sample of subjects with mood disorders with the aim of assessing whether it is possible to identify subgroups of subjects with respect to the classical psychiatric classification and corresponding to different therapies. The same is for suicidal ideation.

\section{Keywords}

Major Depression, Bipolar Disorder, Platelet Fatty Acids, Self Organizing Map, Suicidal Ideation, Adults, Children

\section{Introduction}

Although there is evidence of a continuing effort by the international psychiatric community to refine the diagnosis of mood disorders, to date, the traditional diagnostic criteria are not enough sensitive in identifying patients with bipolar disorder (BD) from those suffering from major depression (MD), in the first diagnosis. Diagnosis remains mostly late and treatments, that may improve symptoms and quality of life, continue to be preceded by interventions which, in addition to not providing adequate relief, often worsen the BD course, increasing the likelihood of inducing rapid cycles or suicidal behaviour (Phillips \& Kupfer, 2013; Mazza, Di Nicola, Janiri et al., 2013).

Differential diagnosis of BD symptoms from other diagnoses has been documented as difficult (Fountoulakis, 2010; Smith et al., 2011; Suppes et al., 2010; Sachs, 1996). Diagnosing BD from MD, psychosis, borderline personality disorders, obsessive-compulsive disorder, etc.) or neuropsychological disorders (cognitive impairment, dementia, etc.) or neuropsychological disorders, has presented challenges. Moreover, manifestations are highly variable not only from patient to patient, but also in the same subject at different stages of the clinical course and in later life.

To overcome this impasse various strategies have been identified and more sensitive and specific assessment tools have been searched for discriminating the BD condition and overcome the delay of an accurate diagnosis has been particularly difficult with MD. The BRIDGE study indicated a first way to go (Angst, Azorin, Bowden et al., 2011), and highlight the strength of some variables such as: mania/hypomania developing during therapy with an antidepressant or other drug, mood lability developing during antidepressant therapy, 2 or more prior mood episodes, and positive family history of mania/hypomania. A debate is essential between the advocates of traditional diagnostic and therapeutic methods and advocates of emerging methods resulting from new discoveries. Major depressive disorder and other related and nonrelated psychiatric conditions are still characterised and defined by descriptive and non-biological criteria, but it is hoped that we can adequately characterise this 
and other psychiatric disorders with the addition of new quantitative approaches.

Cocchi and Tonello have studied platelet membranes of depressed subjects, enlisting profiles of FAs as a possible measure of the membrane status and to determine whether fatty acids could provide indications of diagnostic help between normal subjects and subjects affected by mood disorders. In the first experimental phase, two mathematical tools were identified, a complex one (Self Organizing Map (SOM)) and a simple one (the B2 Index), which will prove valuable not only to define the condition of the Major Depression and Bipolar Disorder, but also to provide the possibility of reasonable inferences about the biological significance of the two molecular mood disorders (Cocchi \& Tonello, 2006; Cocchi, Tonello, \& Cappello, 2006; Cocchi \& Tonello, 2007; Cocchi, Tonello, Tsaluchidu et al., 2008; Cocchi, Tonello, De Lucia et al., 2009a; Cocchi, Tonello, De Lucia et al., 2009b).

The SOM is an artificial neural network that has the ability to put together similar objects and distant different objects using the characteristics of the objects considered.

The B2 index [so named by the authors of the research, (namely, Cocchi and Tonello)] is derived from a mathematical operation that relates the characteristics of molecular weight and melting point of the fatty acids isolated and recognized by the SOM, with the aim of distinguishing the two disorders.

Data studied by means of the Self Organizing Map (SOM) (Kohonen, 2001), the so called "Kohonen Network", suggested three FAs as the main factors in depression and thus as possible strong platelet biomarkers. They are Palmitic Acid (PA), Linoleic Acid (LA) and Arachidonic Acid (AA). The authors speculate that these three FAs, being the majority of the whole FA pattern, might suffice to summarize the whole cell membrane status of viscosity, which may influence the serotonin uptake by cell receptors (Heron, Shinitzky, Hershkowitz et al., 1980; Lee, 1985). Platelets are considered cells with high affinity to neurons; in fact, they have the same embryonic origin of brain and skin (ectoderma) (Evers \& Starr, 2006; Marangos, Campbel, Schmechel et al., 1980; Leonard, 2000). This affinity is primarily related to aspects of serotonin modulation in different psychiatric disorders, particularly MD and BD (Pletscher \& Laubscher, 1980; Da Prada, Cesura, Launay et al., 1988; Camacho \& Dimsdale, 2000; Plein \& Berk, 2001). Those findings have led the scientists to state that platelets can be used as a model to study neurons, defining them as "circulating neurons" or "brain ambassadors".

The main goal of the preliminary experimental results was the realization that the relationship "membranefatty acids" had to be interpreted as a non-linear mathematical model.

Recent evidence suggests, also, that oxidative stress processes might play a relevant role in the pathogenetic mechanisms underlying many major psychiatric disorders, including MD and BD (Ng, Berk, Dean et al., 2008). Patients with BD have significant alteration in antioxidant enzymes (superoxide dismutase), lipid peroxidation and nitric oxide level (Andreazza, Kauer-Sant'anna, Frey et al., 2008; Steckert, Valvassori, Moretti et al., 2010). On the other hand, MD has been associated with lowered concentration of several endogenous antioxidant compounds (vitamin E, zinc, coenzyme Q10) or enzyme (glutathione peroxidase), and with an impairment of the total antioxidant status (Maes, Galecki, Chang et al., 2011; Ozcan, Gulec, Ozerol et al., 2004). Human studies have reported an association between $\mathrm{MD}$ and elevated lipid peroxidation levels such as malondialdheyde (MDA) and F2alpha-isoprostanes (8-iso-PGF2alpha) (Bilici, Efe, Köroğlu et al., 2001; Yager, Forlenza, \& Miller, 2010).

Furthermore, MDA exerts an inhibitory effect on the ligand binding areas of the serotonin receptor, which suggests the existence of a relationship between serotonin metabolism and oxidative stress (Britt, Chiu, Redpath et al., 1992).

Oxidation has been proved to be strictly related to inflammatory processes (Rawdin, Mellon, Dhabhar et al., 2012); indeed, meta-analysis studies have revealed an increased peripheral blood levels of cytokines, interleukin (IL)-6, tumour necrosis factor (TNF)-alpha, and C reactive protein (CRP) in MD patients (Dowlati, Herrmann, Swardfager et al., 2010).

Inflammation appears relevant to BD (Berk, Kapczinski, Andreazza et al., 2011). Available evidence indicates that BD and inflammation are linked through shared genetic polymorphisms and gene expression as well as altered cytokine levels during symptomatic and asymptomatic intervals (Goldstein, Kemp, Soczynska et al., 2009).

The primary objective of this work was to verify if the triplet of FAs indicated by the SOM together with the B2 index was able to recognize the subjects with MD by the subjects with BD. In order to reach this topic, a data base of 3 groups has been built: Major Depression, Bipolar Disorders and Normal Control. Then, the data have been compared with author's historical data and findings obtained in previon children from a 2008 study (Coc- 
chi, Tonello, Cappello et al., 2008). In fact, to the best of our knowledge, a comparison between adults and children with regard of Major Depression and Bipolar Disorders seems to be a big gap within the scientific literature. So that, the authors have tried to shed light on this side of psychopathology.

\section{Subjects and Methods}

The ethical committee of the University of Urbino approved the protocol of the study and informed written consent was given by all participants.

The study was conducted according to the Declaration of Helsinki. The parents of the subjects were given details of the study and they gave informed consent.

\subsection{Recruitment of Subjects}

All volunteer participants were recruited in the judgment of psychiatrists as they presented themselves to the office visit of the "Dipartimento di Salute Mentale", ASUR 4, Fano, Italy. Their personal data were stored and protected at the "Dipartimento di Salute Mentale" of Fano, according to the Italian privacy law (D. Lgs. 196/2003).

All subjects were submitted to a semi-structured interview based on DSM-IV-TR criteria. 21-item Hamilton Depression Rating Scale (HDRS), Hamilton Anxiety Rating Scale (HARS), Clinical Global Impressions Scale (CGI), Symptom Checklist-90 (SCL-90), and Hypomania/Mania Symptom Checklist (HCL-32) were also applied to each patient. Diagnostic agreement among the project interviewers was excellent; indeed, all diagnoses and clinical tests were conducted by psychiatrists according to "Good Clinical Practice Guidelines” to minimize variability.

Psychiatrists have recruited 105 adult patients, than a group of 27 normal controls completed the Data Base:

[(females $=71(68 \%)-$ males $=34(32 \%)$, average age: $50.3 \pm 13.7]$ of whom:

1) Major Depression $[n=40(f=28(70 \%), m=12(30 \%)$, average age: $50.5 \pm 14.7]$

2) Bipolar Disorders [ $\mathrm{n}=65(\mathrm{f}=43(66 \%), \mathrm{m}=22(34 \%)$, average age: $50.2 \pm 13.1]$

3) Normal controls were recruited from laboratory personnel, university staff or their family members. The control group (CTR) comprised 27 apparently healthy volunteers $(\mathrm{M}=17, \mathrm{~F}=10)$ matched for age, sex and socio-cultural condition to pathological subjects.

The subjects were recruited without regard to sex, age, food intake patterns, or pharmacological therapies.

Adult subjects who did not give consent, patients with a diagnosis other than an affective disorder and subjects in their first clinical episode were excluded. The study was double blind.

\subsection{Procedures}

\subsubsection{Blood Sampling}

Ante cubital venous blood was drawn between 8:00 AM and 9.30 AM from fasting subjects on the day of recruitment at the "Dipartimento di Salute Mentale" of Fano, Italy. Blood samples were collected into EDTA Vacutainer $^{\circledR}$ tubes and immediately processed by centrifugation to obtain plasma, platelet and lymphocyte samples. Analyses were carried out in double-blind.

The IRB requirement has been obtained by the Committee of the Marche Region who had approved the research project.

\subsubsection{Platelet Isolation}

Platelet rich plasma (PRP) was obtained by centrifuging whole blood at $800 \mathrm{rpm}$ for $20 \mathrm{~min}$ at $4^{\circ} \mathrm{C}$; a further centrifugation of PRP at $5000 \mathrm{rpm}$ for $5 \mathrm{~min}$ at $4{ }^{\circ} \mathrm{C}$ allowed to obtain the platelet pellet, which was first resuspended in $500 \mu \mathrm{l}$ of physiological saline and then centrifuged at $5000 \mathrm{rpm}$ for $5 \mathrm{~min}$ at $4^{\circ} \mathrm{C}$ ("Beckman Coulter" microfuge centrifuge R-USA). Pellets were stored at $-80^{\circ} \mathrm{C}$ until analysed.

\subsubsection{Platelet Membrane Fatty Acid Composition (Folch, Less, \& Sloane Stanley, 1957)}

The platelet pellet was shaken for 20 minutes with $100 \mu \mathrm{l}$ of $2 \mathrm{M} \mathrm{KOH}-\mathrm{CH}_{3} \mathrm{OH}$ solution to convert FAs in methyl esters. The FA methyl esters were then extracted with $100 \mu \mathrm{l}$ of n-hexane and stirred with a vortex for 20 seconds. After the separation of the two phases, the hexane layer containing FA methyl esters was transferred into a clean tube. The residue phase containing methanol was extracted again with $100 \mu \mathrm{l}$ of n-hexane and 
stirred with a vortex for 20 seconds. The hexane layer containing FA methyl esters was added to the previously collected one and evaporated to dryness under nitrogen.

A gas chromatograph (Agilent $6890 \mathrm{~N}$, USA) coupled with double focalization high resolution Jeol JMSGC-Mate II (Japan) in full scan mode equipped with Jeol Software was used for GC/MS confirmation of FA methyl esters. Chromatographic separation of FA methyl esters was performed using a Supelcowax 10 (0.25 mm i.d. $\times 30 \mathrm{~m}$ length, $0.25 \mu \mathrm{m}$ film thickness). The oven temperature was programmed as follows: $100^{\circ} \mathrm{C}$ for $3 \mathrm{~min}$, ramped at $20^{\circ} \mathrm{C} / \mathrm{min}$ to $165^{\circ} \mathrm{C}$, ramped at $12^{\circ} \mathrm{C} / \mathrm{min}$ to $270^{\circ} \mathrm{C}$. The final isotherm was of $270^{\circ} \mathrm{C}$ for 5 minutes for clean column. The carrier gas was helium with a constant flow of $1 \mathrm{ml} / \mathrm{min}$. The injector temperature was $260^{\circ} \mathrm{C}$ and the interface temperature was $270^{\circ} \mathrm{C}$. Injection volume was $1 \mu \mathrm{l}$ with 3 minutes of delay time in splitless mode.

The following FAs were separated during a run time of 20 minutes: Palmitic acid (C16:0), Stearic acid (C18:0), Oleic acid (C18:1, n-9), Linoleic acid (C18:2, n-6), and Arachidonic acid (C20:4, n-6). Each FA was identified by comparing its fragmentation pattern with the mass spectrometer database. The content of individual FA in platelet membranes was expressed as percentages of the total FAs.

\subsection{Mathematical Method}

\subsubsection{Self Organizing Map (SOM)}

The SOM was created by Teuvo Kohonen as an unsupervised competitive-learning network algorithm. The central property of the SOM performed in the current paper is that it forms a nonlinear projection of a 3-dimensional data manifold on a regular, 2D grid. The data items are vectors, the components of which are the amount of AP, AL and AA, of each subject. The SOM grid can be used as a groundwork on which each of the vectors can be displayed separately. This kind of combined display has been found very useful for the understanding of the mutual dependencies between the variables, as well as of the structures of the data set. In the context of this definition, a manifold refers to a topological space with well-defined mathematical properties. In the display, the clustering of the data space as well as the metric-topological relations of the data items, are clearly visible. A particular strength of the SOM displays lies in enabling relevant information to be "found" rather than "searched for”.

\subsubsection{On the Non-Manipulability of the SOM Built for the Classification of the Psychiatric Subjects}

Let's suppose we want to build a fake SOM, that is, a SOM driven by us according to a desired result. We should be very lucky, in fact we should guess:

1200 particular numbers (starting weights). By the way, really, it is impossible to know how they could be chosen in order to obtain a particular result.

Above all, we should find a particular order of data that, because of an unknown reason, lead to a very particular result. In our case, we have a data base of 144 Subjects (84 depressive and 60 normal). This means that there are $144 !=5.5503 \times 10,249$ combinations. A training process takes about 4 minutes. So, about $4.224 \times$ 10,242 centuries are needed to check all possible results.

\subsubsection{B2 Index}

The B2 index was obtained from the summation of the percentages of each fatty acid (selected by SOM) multiplied by its melting point and divided by its molecular weight, obtaining an indirect expression of membrane viscosity, which induces to identify it with the neuron membrane viscosity, as described (Cocchi, Tonello, \& Gabrielli, 2012).

\subsubsection{Plasma and Platelet Serotonin Levels}

Serotonin levels were assessed in each patient both in platelet pellets and in poor platelet plasma samples. Quantitative determination of serotonin levels was performed by enzyme immunoassay using a commercially available kit from IBL International (Hamburg, Germany), following the instructions furnished by the manufacturer.

\subsubsection{Oxidative Stress and Inflammation Markers}

Carotenoids (lutein, lycopene, beta carotenes), vitamins A (retinol) and vitamin E (alpha tocopherol) were measured in plasma samples by reversed-phase HPLC after sample deproteinization with ethanol and extraction 
with hexane as previously described (Aebischer, Schierle, \& Schuep, 1999). Quantitative determination of oxLDL (oxidized low-density lipoprotein), IL-6 (Interleukin-6), TNF- $\alpha$ (Tumor Necrosis Factor- $\alpha$ ), LTB4 (Leukotriene B4), PGE2 (Prostaglandin E2), and TXB2 (Tromboxane B2) was performed by enzyme immunoassays using commercially available kits from Biomedica (Wien, Austria) and Enzo Life Sciences (Lausen, Switzerland), following the instructions furnished by the manufacturer. Homocysteine, CRP (C Reactive Protein), and ESR (Erythrocyte Sedimentation Rate) were evaluated by routine laboratory protocols at the "Santa Croce” Hospital in Fano.

\subsubsection{Statistics and Data Processing}

Descriptive analysis included mean and standard deviation (SD). Normality of the distribution was evaluated by the Kolmogorov-Smirnov test. The statistical analysis was carried out using the one-way analysis of variance (ANOVA) for normal distributions or by the Kruskal-Wallis test for not normal distributions. The Tukey test was used as post-hoc ANOVA test for multiple comparisons. Probability values of $<0.05$ were accepted. Statistics were obtained using WinSTAT (R. Fitch Software).

\section{Results}

After selection, 105 subjects were included:

Among MD, 19 patients were on treatment with serotoninergic drugs, 10 with non-serotoninergic and 11 received no therapy. Among BD, 28 patients were on treatment with serotoninergic drugs, 14 with non-serotoninergic and 23 received no therapy.

The control group (CTR) comprised 27 apparently healthy volunteers $(M=17, \mathrm{~F}=10)$ matched for age, sex and socio-cultural condition to pathological subjects.

Controls, assessed by a semi-structured psychiatric interview, were free of psychiatric illness or, at least, a psychiatric condition was not expressed. None of them was using anti-depressant therapy. For these subjects the B2 index was considered, and they were classified according to negative or positive values. In short, the control group was selected among subjects with positive and negative B2 index, without expression of psychopathology and without antidepressant assumption. Only in this way, researchers would be able to identify biomarker differences compared to drug treatment.

\subsection{Fatty Acid Composition of Platelet Membrane}

The A composition of platelet membrane is shown in Table 1.

Five major FAs can be detected by GS/MS technique: the most abundant is oleic acid, while the less is linoleic acid. Significant differences were detected in platelet FA composition among patients and controls. In detail, MD patients presented increased levels of arachidonic acid and reduced levels of palmitic acid as compared to CTR. On the contrary, BD patients had increased levels of palmitic acid and reduced levels of arachidonic acid than CTR. Consequently, MD and BD subjects differentiated in platelet FA composition in relation to palmitic and arachidonic acid level; in addition, MD and BD groups also presented significantly different levels of linoleic acid (Figure 1).

\subsection{Platelet Membrane Viscosity Index}

The platelet membrane viscosity index (B2) for each group of subjects is reported in Table 2. By uploading palmitic, linoleic and arachidonic acid content into the SOM, mean B2 values equal to 0.205 for CTR, -0.329 for MD and 0.808 for BD were obtained. Values were statistically different from each other $\left(p=1.4 \times 10^{-13}\right.$, ANOVA). All depressed patients presented a negative B2 index while all bipolar patients had a positive index. CTR subjects showed both positive and negative indexes (from -0.93 to 2.16); in detail, controls with a negative index had a mean B2 value equal to -0.369 , while those with a positive index a mean B2 value equal to 0.650 .

\subsection{Evidence of the SOM in Classifying Depressive and Bipolar Subjects}

Platelet fatty acids (PA, LA, AA) results obtained from apparently normal subjects and from subjects generically classified with Mood Disorders (psychiatric diagnosis) were analysed through the use of a Self Organizing Map (SOM), as described in previous reports (Cocchi, Tonello, \& Cappello, 2006; Cocchi \& Tonello, 2006; Cocchi, 
Table 1. Fatty acid composition of platelet membrane in controls and MD and BD patients. Data are normally distributed and expressed as mean \pm SD.

\begin{tabular}{ccccc}
\hline Fatty acid (\%) & CTR & MD & BD & $\boldsymbol{p}$ value (ANOVA) \\
\hline Palmitic acid (C16:0) & $18.09 \pm 1.69$ & $17.12 \pm 0.75^{\mathrm{a}}$ & $19.83 \pm 1.90^{\mathrm{a}, \mathrm{b}}$ & $1 \times 10^{-13}$ \\
Stearic acid (C18:0) & $19.85 \pm 2.18$ & $18.91 \pm 0.99$ & $19.41 \pm 1.84$ & 0.089 \\
Oleic acid (C18:1) & $28.05 \pm 2.15$ & $27.67 \pm 1.19$ & $28.37 \pm 2.30$ & 0.219 \\
Linoleic acid (C18:2) & $8.88 \pm 1.69$ & $9.58 \pm 1.87$ & $8.41 \pm 2.20^{\mathrm{b}}$ & 0.018 \\
Arachidonic acid C20:4) & $25.13 \pm 2.33$ & $26.72 \pm 1.03^{\mathrm{a}}$ & $23.97 \pm 2.62^{\mathrm{a}, \mathrm{b}}$ & $4 \times 10^{-8}$ \\
\hline
\end{tabular}

${ }^{\mathrm{a}}$ Significantly different from CTR; ${ }^{\mathrm{b}}$ Significantly different from MD.

Table 2. Platelet’s membrane viscosity (B2 index) in controls and MD and BD patients. Data are expressed as mean (range).

\begin{tabular}{ccccc}
\hline Platelet viscosity & CTR & MD & BD & $p$ value (ANOVA) \\
\hline \multirow{2}{*}{ B2 index } & 0.205 & $-0.329^{\mathrm{a}}$ & $0.808^{\mathrm{a}, \mathrm{b}}$ & $1.4 \times 10^{-13}$ \\
& $(-0.93 / 2.16)$ & $(-1 /-0.011)$ & $(0.01 / 4.1)$ & \\
\hline
\end{tabular}

${ }^{\mathrm{a}}$ Significantly different from CTR; ${ }^{\mathrm{b}}$ Significantly different from MD.

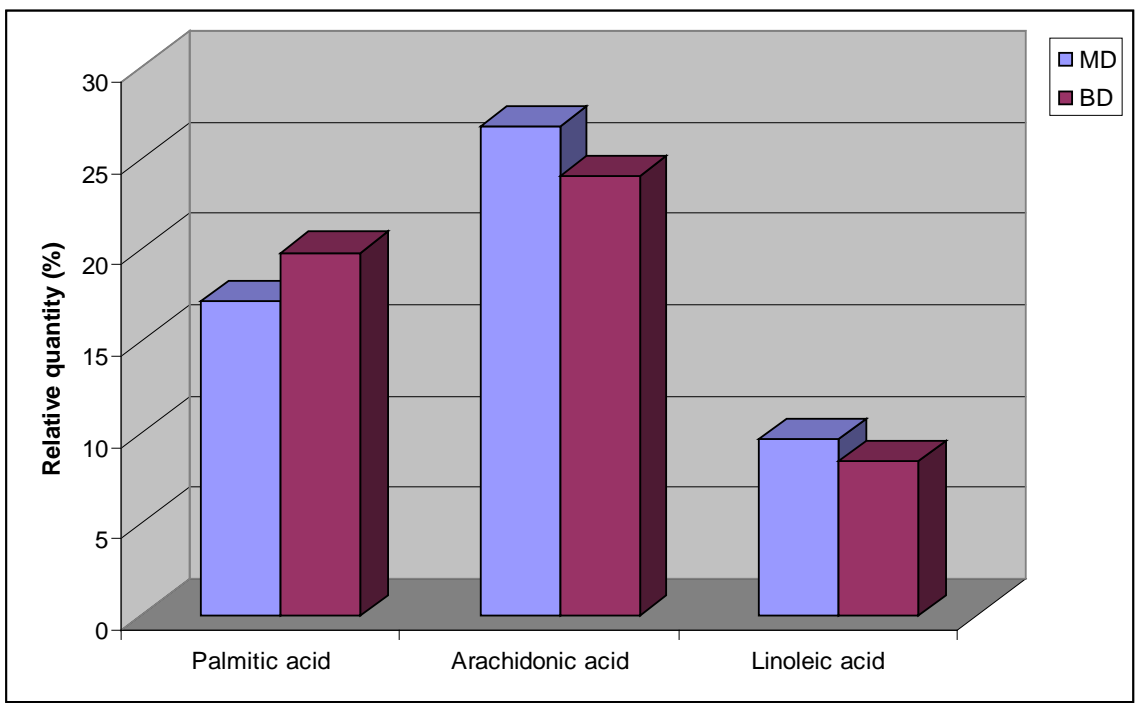

Figure 1. Platelets fatty acids levels in MD and BD.

Tonello, Tsaluchidu, \& Puri, 2008; Cocchi \& Tonello, 2010b).

Figures 2-4. Distribution of normal and pathologic cases [first experiment (29)] over the SOM. In Figure 3 and Figure 4 the two groups have been clustered to better show the distribution.

Three main areas have been obtained:

1) Normal subjects area (a-only green dots);

2) Depressive and Bipolar subjects area (b-only red dots);

3) Mixture of both (c-red and green dots).

The third area was split into two other areas (yellow and orange) according to the major density of normal (yellow) and pathological (orange) subjects.

The combination of the SOM and of the B2 index gave the following results (Figures 5-8).

A new SOM was made to verify if the same triplet of fatty acids was able to confirm the separation between $\mathrm{MD}$ and $\mathrm{BD}$, thus enabling its use for future diagnostic classifications.

The result was showed in (Figure 7) and has been compared with the histogram of the B2 index to verify the correspondence (Figure 8).

The first result was that the SOM properly distinguished and recognized the differences between the two groups of subjects (MD and BD). In Figure 7, the blue area refers to BD, while the brown to MD. The second 


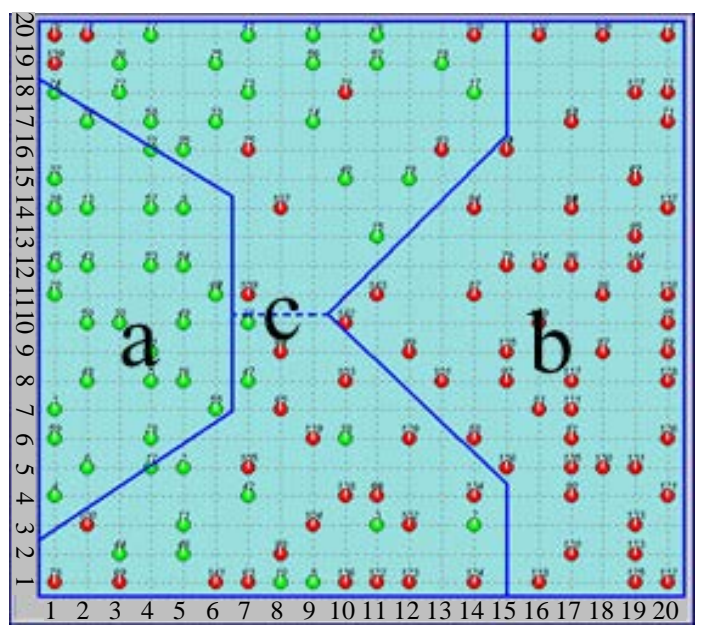

Figure 2. Distribution of all cases of the first experiment (first SOM).

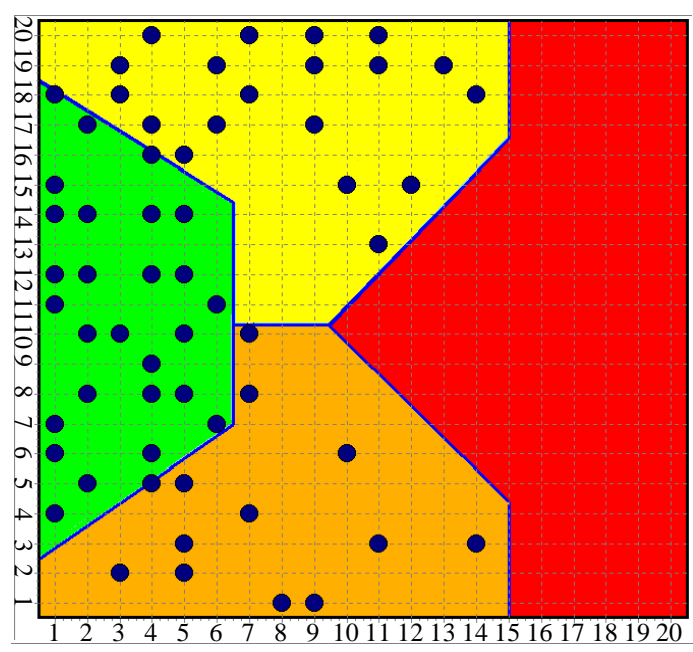

Figure 3. Distribution of normal subjects over the SOM.

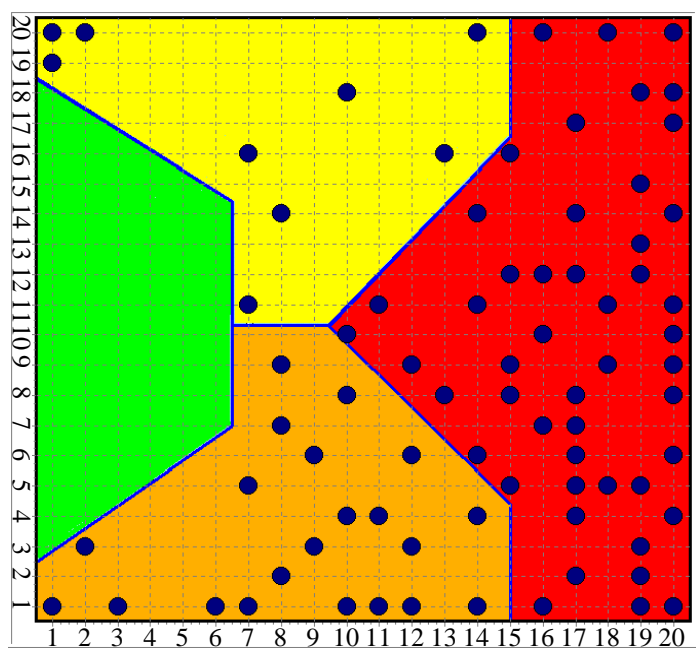

Figure 4. Distribution of depressive subjects over the SOM. 


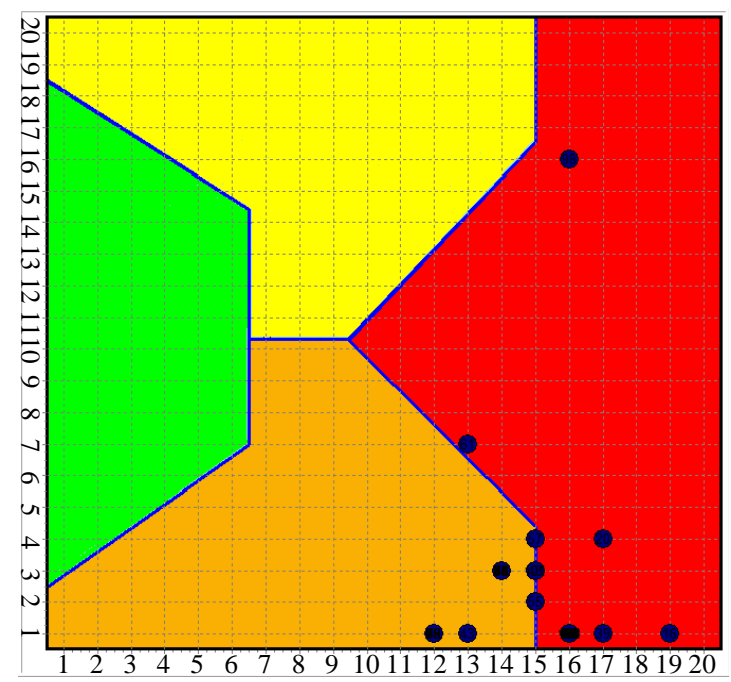

Figure 5. Distribution of the MD and BD subjects (new experiment) over the first SOM built.

\begin{tabular}{|c|c|c|c|c|c|c|c|c|c|c|c|c|c|c|c|c|c|c|c|c|}
\hline 20 & 2.24 & 1,17 & 1,92 & 1,89 & 1,92 & 2,04 & 2,11 & 1.85 & 1.68 & 1.59 & 1.58 & 1.56 & 1.64 & 1,60 & 1,30 & 0.99 & 0.50 & 0.02 & -0.49 & -1.02 \\
\hline 19 & 1,92 & 1,94 & 2,25 & 2,08 & 1,98 & 1.98 & 2,00 & 1.71 & 1,53 & 1.44 & 1,37 & 1.53 & 1,70 & 1.54 & 1,32 & 0.96 & 0,48 & 0.10 & 0.12 & -0.21 \\
\hline 18 & 2.4 & 2.28 & 2.21 & 2,20 & 2.11 & 2.00 & 1.91 & 1.83 & 1.55 & 1.34 & 1.36 & 1.50 & 1.51 & 1,33 & 1.29 & 0.62 & 0.39 & 0,36 & 0.36 & 0.44 \\
\hline 17 & 2.36 & 2,37 & 227 & 2,19 & 2.22 & 2,12 & 2,04 & 1.85 & 1.77 & 1.55 & 1.39 & 1.32 & 1,31 & 1,30 & 1,23 & 0.49 & 0.39 & 0.38 & 0.43 & 0.51 \\
\hline 16 & 3,03 & 2.57 & 2.35 & 250 & 2,31 & 2,18 & 2,09 & 2.03 & 1.71 & 1.47 & 1.29 & 1.23 & 1.31 & 1.27 & 1.25 & 0.97 & 0.40 & 0.43 & 0.46 & 0.47 \\
\hline 15 & 3,21 & 3,19 & 2,59 & 2,47 & 2,53 & 239 & 2,21 & 2,41 & 1.67 & 1.40 & 1.27 & 1.14 & 1.22 & & 1,22 & 0.72 & 0,53 & 0.47 & 0.47 & 0.15 \\
\hline 14 & 3,13 & 3,24 & 2.76 & 2,55 & 2,79 & 2.7 겨 & 2,55 & 2,57 & 2,27 & 1.36 & 1.15 & 1.13 & & & 1.08 & 0.55 & 0.54 & 0.48 & 0.11 & -0.49 \\
\hline 13 & 2.99 & 3.05 & 2.53 & 2.44 & 2,53 & 2.41 & 2,42 & 2.53 & 2,17 & 1.17 & 1.09 & & & 1,01 & 0.67 & 0.49 & 0.51 & 0,18 & -0.01 & -0.24 \\
\hline 12 & 2,91 & 2.83 & 2.35 & 2,28 & 2.34 & 2,14 & 2.01 & 2,06 & 1.97 & 1.63 & & & & 0,67 & 0.60 & 0,43 & 0.48 & 0,12 & -0.21 & -0.27 \\
\hline 11 & 2,39 & 2,54 & 2,44 & 2.31 & 2,22 & 2,1 . & 1.93 & 1.97 & 2.22 & 2.20 & & & 0.79 & 0.73 & 0.63 & 0.48 & 0.31 & 0,07 & $-0,18$ & -0.38 \\
\hline 10 & 2,49 & 2,55 & 2,45 & 2,41 & 2,28 & 2,1 & 2,01 & 1,97 & $\overline{2}, 27$ & 2,40 & & 1.68 & 1.07 & 0,68 & 0,55 & 0.50 & 0,35 & 0,05 & -0.30 & -0.42 \\
\hline 9 & 2.83 & 2,78 & 2,54 & 2,52 & 2,46 & 2.29 & 2,05 & 1,91 & 2.02 & 2.21 & & & 1.37 & 0.69 & 0,58 & 0.51 & 0.22 & 0,04 & -0.24 & -0.40 \\
\hline 8 & 3.28 & 3,10 & 2,78 & 2.53 & 2.57 & 2.4 & 2.23 & 2.03 & 2,01 & 2.05 & 1.91 & & 1.23 & 0.87 & 0.58 & 0,51 & 0.32 & 0.19 & $-0,41$ & -0.57 \\
\hline 7 & 3,45 & 3,33 & 2.88 & 2,69 & 2.57 & 250 & 2.22 & 1.93 & 1.97 & 1.82 & 1.66 & 1.38 & & 0.85 & 0.63 & 0.64 & 0,38 & 0,32 & -0.37 & -0.51 \\
\hline 6 & 3,78 & 3,74 & 3.07 & 2,86 & 206 & 2,59 & 2,43 & 2.06 & 2,07 & 1.54 & 1.50 & 1,40 & & & 0.78 & 0.44 & 0.26 & 0,08 & $-0,36$ & -0.46 \\
\hline 5 & 4,05 & 3,80 & 3.40 & 5,13 & 2.93 & 2.76 & 2.54 & 2.49 & 1.99 & 1.81 & 1.83 & 1.57 & 1.12 & & 0.75 & 0.33 & -0.05 & -0.05 & -0.35 & -0.54 \\
\hline 4 & 4.60 & 4.20 & 3.75 & 3,18 & 3,18 & 2,91 & 2,85 & 2.71 & 2,20 & 1.99 & 1.89 & 1.85 & 1.23 & 1.01 & 0,91 & 0.10 & -0.03 & -0.14 & -0.70 & -0.80 \\
\hline 3 & 4 & 4.20 & 4,18 & 3,68 & 3.45 & 3,36 & 2,90 & 2,69 & 2,42 & 2,09 & 1.93 & 1.77 & 1.51 & 1.34 & 1.18 & -0.01 & -0.18 & -0.82 & -1.07 & -1.01 \\
\hline 2 & 7.10 & 4,47 & 4.14 & 3,06 & 3.63 & 3.40 & 3.34 & 2.91 & 2.75 & 2.43 & 2,08 & 2.05 & 1.66 & 1.19 & 0.73 & -0.14 & -0.20 & $-1,00$ & -1.03 & -1.95 \\
\hline 1 & 8.23 & 6.58 & 4.26 & 3,98 & 3.38 & 3.08 & 3.89 & 3.07 & 2.90 & 2,50 & 2,16 & 2.34 & 1.72 & 1.03 & 0,50 & -0.03 & $-0,19$ & $-1,48$ & $-1,79$ & -2.84 \\
\hline & 1 & 2 & 3 & 4 & 5 & 6 & 7 & 8 & 9 & 10 & 11 & 12 & 13 & 14 & 15 & 16 & 17 & 18 & 19 & 20 \\
\hline
\end{tabular}

Figure 6. Distribution of the B2 index and overlapped the SOM clusters (dark lines). The blue line delimits the negative indexes (MD).

result was that there was an intermediate area, coloured in purple. Many SOM tests have been made and they always create an intermediate zone. The SOM answer was not the same of the B2 index which recognize the subjects also in a narrow range of the index and that at zero, set a net threshold.

This is an independent test (which uses new data on depression and who works in a different way) that the three fatty acids are associated with psychiatric disorders.

\subsection{Serotonin Levels}

Plasma and platelet serotonin levels are reported in Table 3. Significant differences were found among patients and controls; indeed, MD and BD groups presented lower levels of both plasmatic and platelet serotonin than CTR subjects. 


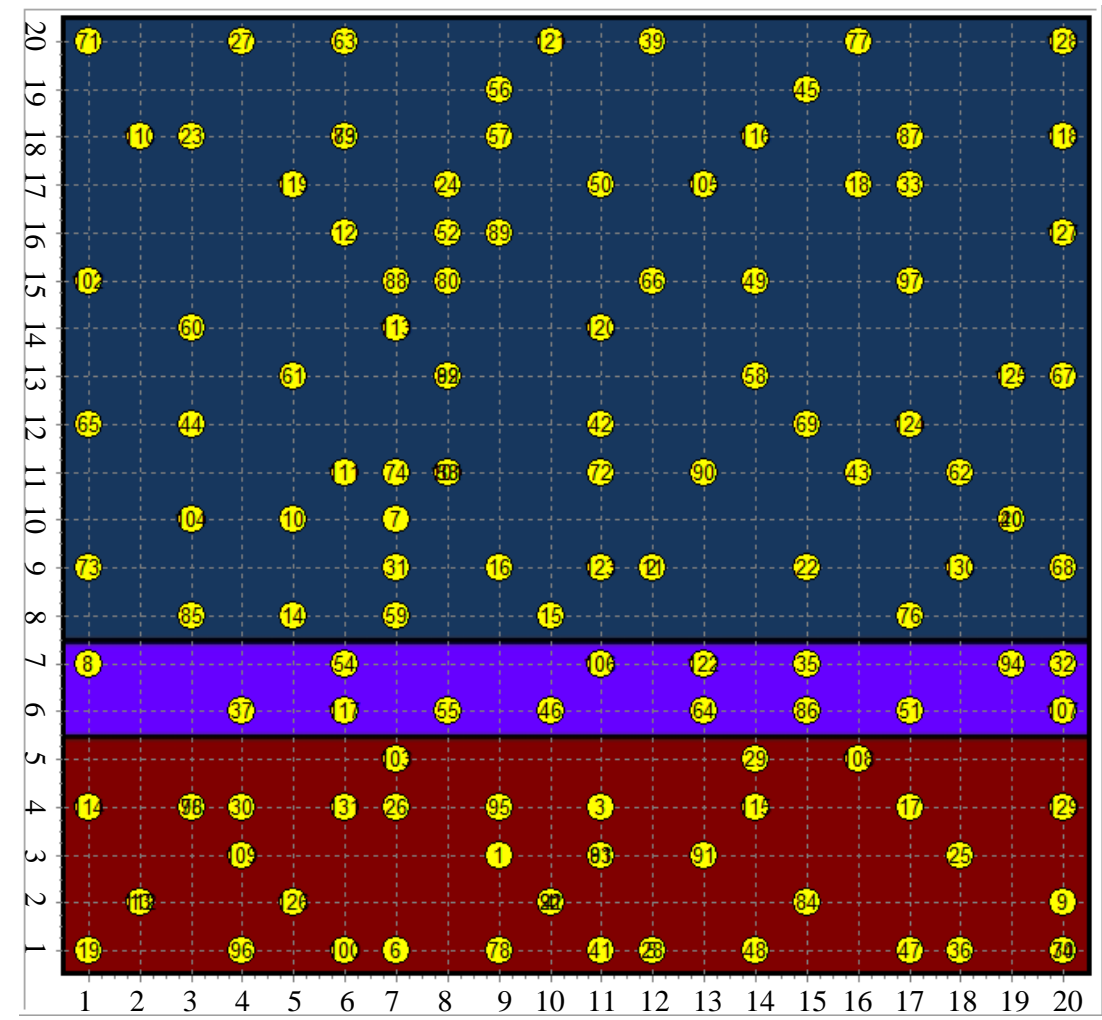

Figure 7. The new SOM recognize the MD (brown area) and the BD (blue area).

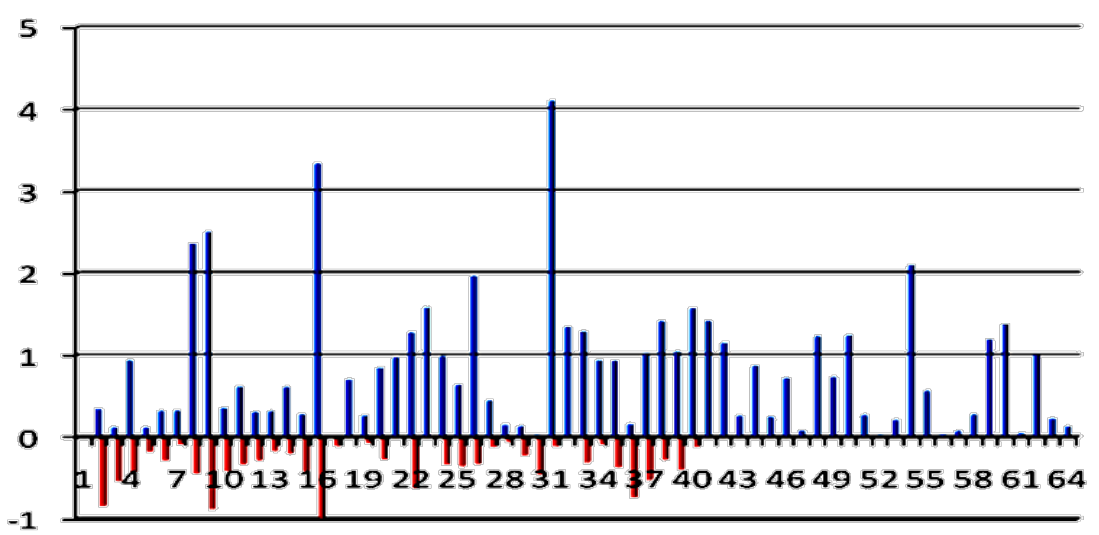

Figure 8. Histogram of the $\mathrm{B} 2$ index (blue $=\mathrm{BD}$ and red $=\mathrm{MD}$ ).

Table 3. Plasma and platelet serotonin levels in CTR subjects and in MD and BD patients. Data are not normally distributed and are expressed as mean (range).

\begin{tabular}{ccccc}
\hline SEROTONIN & CTR & MD & BD & $p$ value (Kruskal-Wallis) \\
\hline Plasma (ng/ml) & $7.2(1.2-22.0)$ & $5.1(0.2-24.0)$ & $4.5(0.3-24.9)$ & 0.005 \\
Platelets $(\mathbf{n g} / \mathbf{1 0}$ platelets) & $561(233-1472)$ & $290(1-1129)$ & $252(1-1454)$ & 0.00005 \\
\hline
\end{tabular}

Within the CTR group, subjects with a positive platelet membrane viscosity index had significantly higher levels of platelet serotonin than those with a negative index (557 vs. $433 \mathrm{ng} / 10^{9}$ platelets, $p<0.05$ Mann-Whitney U-test) (Figure 9), confirmed that increased cell membrane viscosity correlated with increased serotonin uptake. 


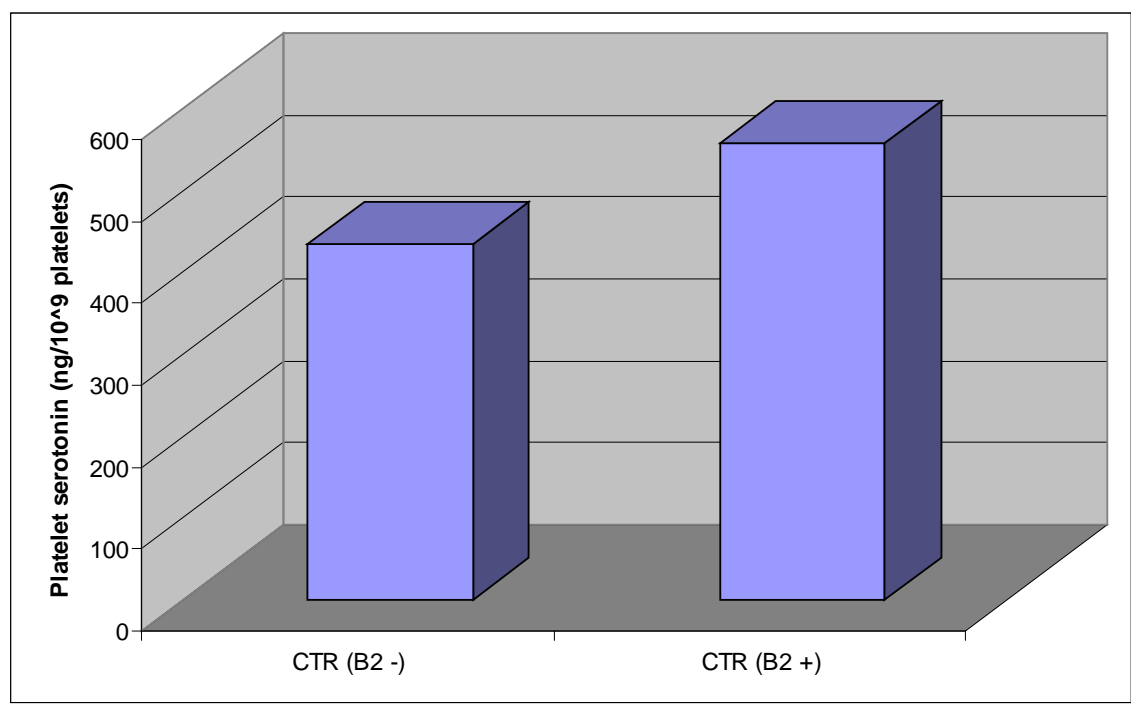

Figure 9. Increased platelet serotonin levels in CTR subjects showing a positive membrane viscosity index $(\mathrm{B} 2+)$ as compared to subjects with a negative index $(\mathrm{B} 2-)(p<$ 0.05 Mann-Whitney U-test).

\subsection{Comparison between Adult and Children}

We used the data base of a previous research on children ( $n=112$ age: $10.5 \pm 2.7-m \pm S D$ ), not subjected to psychiatric diagnosis, in order to verify the distribution of the children themselves, over the SOM, through the triplet of the fatty acids identified by the mathematic function (Cocchi, Tonello, Tsaluchidu et al., 2008). Comparing adults (Figure 10) and children (Figure 11) a first consideration was possible: none of the children is in the red area and because all the subjects with Major Depression are placed in a specific part of the red area (see Figure 5 and Figure 6), it seems not possible to attribute to any child the condition of MD but, to some of them, of $\mathrm{BD}$. One possible explanation is that the children's platelet fatty acids profile has a very high concentration of stearic acid, to compensate the lower cholesterol of the membrane as described in young organisms (Nicholson \& Ferreira, 2009) and, as a result, the children's platelet membranes are highly saturated with increased viscosity. This corresponds to a higher uptake of serotonin with respect to the MD condition (Cocchi, Tonello, \& Gabrielli, 2012; Lee, 1985; Heron, Shinitzky, Hershkowitz et al., 1980). The concept of cell membrane viscosity, especially that of the neuron and platelet, also responsible of the central government of that bio-molecular intracellular complex, called interactome (the whole array of molecular interactions that take place in an organism and allow the cascade of regulatory molecules including the mechanism of action of enzymes and metabolic reactions) and of its influence on the ion channels, is often missed (Cocchi, Gabrielli, Tonello et al., 2010) in its involvement in many psychiatric conditions. Under this evidence and according to the classification of the children over the SOM we hypothesize that it is very difficult to have a classic condition of Major Depression in children.

\subsection{SOM and "Suicidal Ideation"}

Distributing the adult subjects (MD e BD) over the first SOM (Figure 4) we noticed that some of them were placed in the area which corresponds to the minimum level of Linoleic Acid (Figure 12), the same for the children (Figure 11).

Of the whole group of patients, 8, diagnosed with "suicidal ideation" (psychiatric evaluation), have been clustered over the SOM and compared to children with the same platelets' molecular characteristic of the "adult suicidal ideation" (Figures 13-15).

As it is possible to see in Figure 14, the "adult suicidal ideation" (7 bipolar, 1 major depression) range between the position 13 and 16 of the abscissa (and $\mathrm{Y}<4$ ) clearly forming a cluster. We have identified this area as a cluster of "suicidal ideation". Because of the precision of the SOM, it seems excluded the position 12 for the children, so, the children, really corresponding to the "adult suicide" position, are two. 


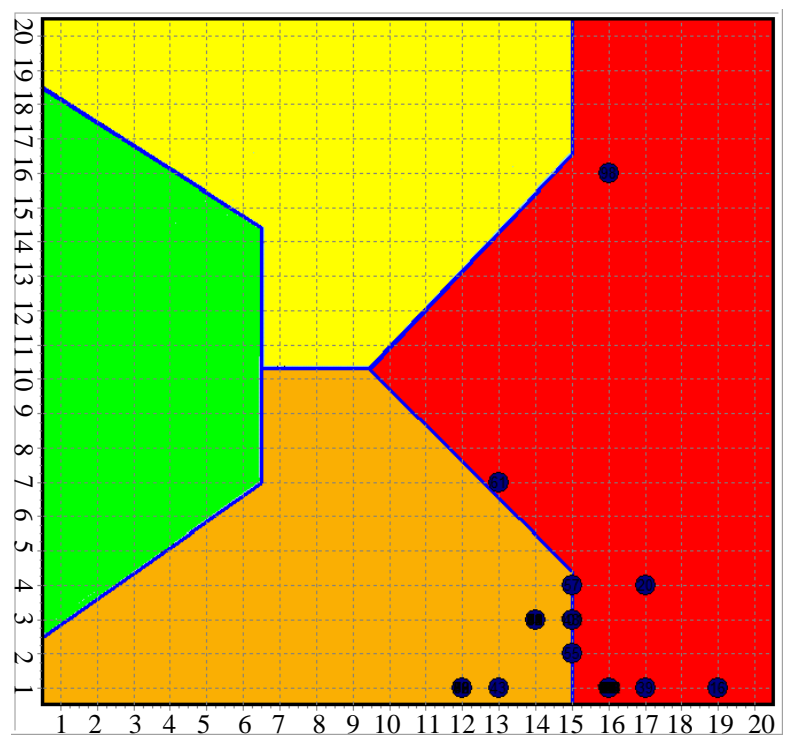

Figure 10. Distribution of the MD and BD subjects over the SOM.

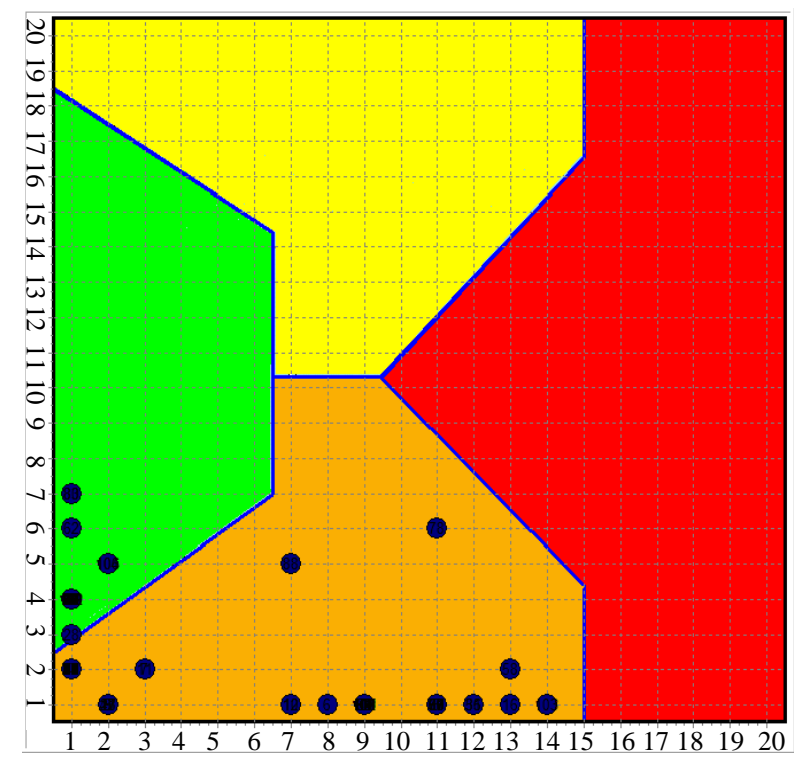

Figure 11. Distribution of all children's over the SOM.

We tried to study the "suicide area" of the SOM in order to find common feature among people with suicide thought or attempt, according to the SOM itself. We have focused our attention at the level of the three Fatty Acid and found that the "suicide area" of the SOM corresponds to the lowest value of the LA, which seems the critical aspect in "suicide ideation", as showed in Figure 6.

We know that the BD is the psychiatric condition with a higher risk of suicide (Mathews, Richards, \& Niciu, 2013) and it is also likely that it is not possible to investigate and highlight the real risk without the use of biological markers.

Some risk factors have been identified (Undurraga, Baldessarini, Valenti et al., 2012; Bellivier, Yon, Luquiens et al., 2011) as well as different ways to highlight biological markers of suicide have been tried (Turecki, Ernst, Jollant et al., 2012; Pandey \& Dwivedi, 2012; Pandey, 2011; Mann \& Currier, 2010; Donati, Dwivedi, Roberts et al., 2008; Mann, 2003; Turecki, 2001; Stanley \& Stanley, 1989; Stanley et al., 1986).

Even with strong implications for plausibility these attempts have not been successful, up to now, to identify 

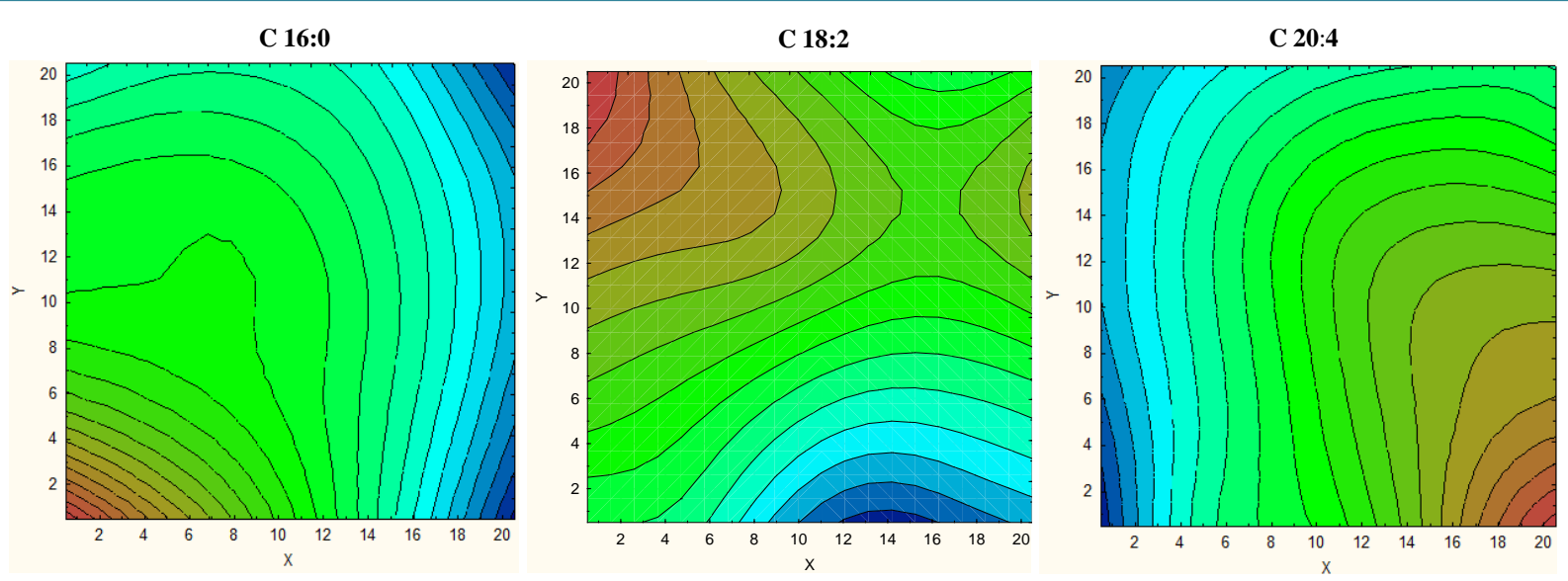

Figure 12. Level curve of PA, LA, AA over the SOM. The picture show the level of LA (C18:2) in a level curve representation. Higher levels of LA are brown coloured; middle levels are green while lower levels are blue. The so called "suicide" area corresponds to the minimum value of LA. Is C18:2, the main actor of "the fine tuning"?

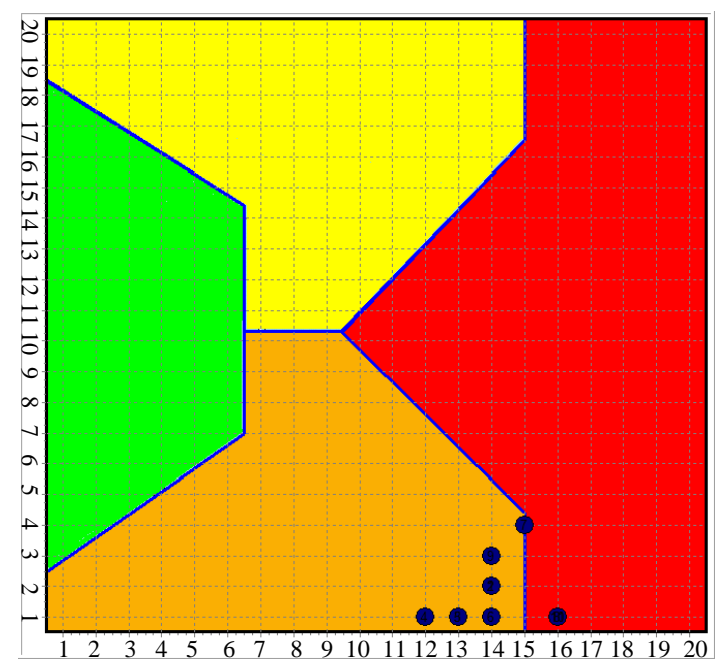

Figure 13. Distribution of all "adult suicide” together with children.

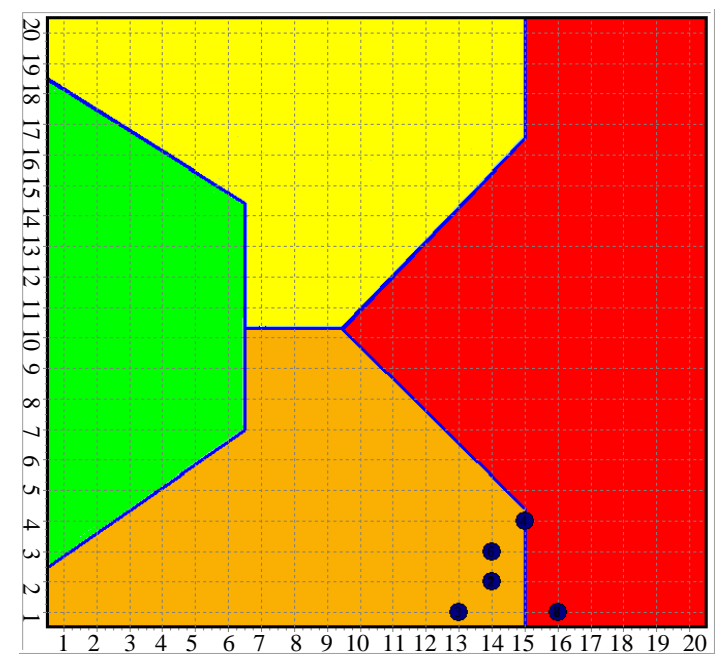

Figure 14. Distribution of "adult suicide". 


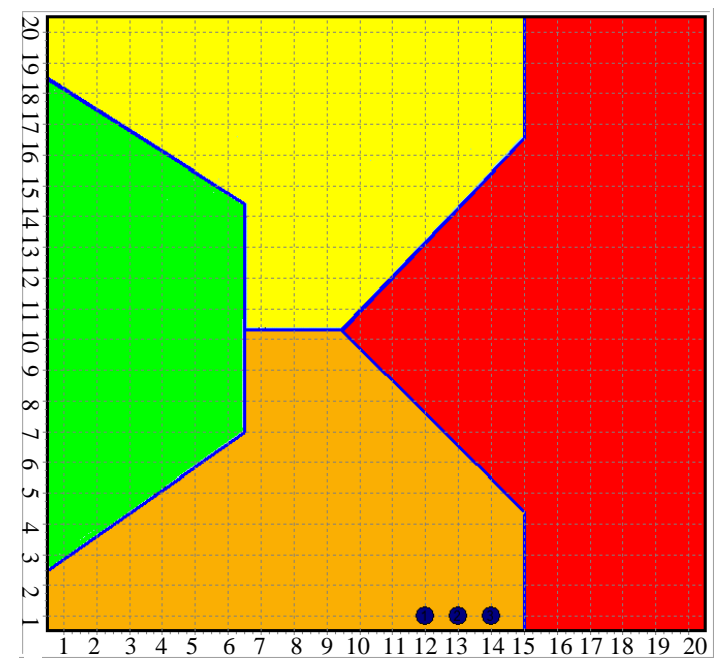

Figure 15. Distribution of children with molecular similarity to "adult suicide".

classification tools that may allow early interventions for prevention.

Despite the awareness that the SOM cannot be manipulated, and considering its very high degree of diagnostic precision, but in view of the fact that we have not submitted the children, recruited in a common medical department, to psychiatric evaluation for the complexity of the ethical reasons, we are aware that these issues need to be further explored. This may allow alerting the psychiatrist in front of the not infrequently, dramatic, child's predisposition to suicide.

The index of membrane fluidity is stable in the same subject over time. A condition of greater or lesser clinical compensation corresponds to variations which are not substantial and never register transition from a positive value to a negative or vice versa. Clinical improvement appears to correspond to an increase in the B2 index of the membrane, i.e. increasing the viscosity.

\subsection{Serotonin and Linoleic Acid}

Because of the Linoleic Acid features we found in suicidal ideation, we investigated whether there might be a correlation between platelet's serotonin concentration and fatty acids.

The study carried out (Figure 16) showed that there was a significant correlation $(p<0.05)$ between fatty acids and platelet serotonin for one of them, just the LA, in the case of lower values of platelet serotonin $(<100)$.

The group of subjects who has a level of serotonin $<100$, is characterized by a statistically significant difference with the lower level of linoleic acid than did those with higher levels of serotonin.

To increase the level of certainty of the previous statement we wanted to verify the result by comparing two groups of subjects, equipotent. We operated in the following way: 2 groups were formed of the same numerosity (60 subjects each). A group containing the 60 lowest levels of serotonin and another that has the highest values. Repeating the analysis, it was found again significance only for the level of linoleic acid. In conclusion, it is confirmed that, at least in the sample considered, low levels of serotonin are associated with low levels of linoleic acid $(p<0.05)$, the only statistically significant.

This result would be consistent with the finding that a reduction of serotonin corresponds to the physicochemical state (viscosity) of the platelet membrane as described, for other aspects, by Engelberg, 1992.

\subsection{Biomarkers of Oxidative Stress and Inflammation}

The biochemical parameters related to oxidative stress assessed in CTR, MD and BD subjects were reported in Table 4.

As regards the antioxidant defense system, significant differences $(p<0.05)$ were observed in carotenoid plasma levels among the three groups (see Table 4). In fact, in subjects with MD and BD, carotenoids were significantly lower than in CTR, with reductions from $20 \%$ (lutein and lycopene) to $40 \%$ (beta carotene). No sig- 
nificant differences were observed in vitamin A and E plasma levels among the three groups. As regards the markers of oxidative damage, significant differences were observed in oxLDL and homocysteine plasma levels among the three groups. In particular, MD subjects presented a significant increase in the levels of both oxLDL and homocysteine as compared to CTR and BD subjects.

In relation to inflammation (Table 5), a relevant accumulation of TNF-alpha, LTB4, PGE2 and TXB2 was

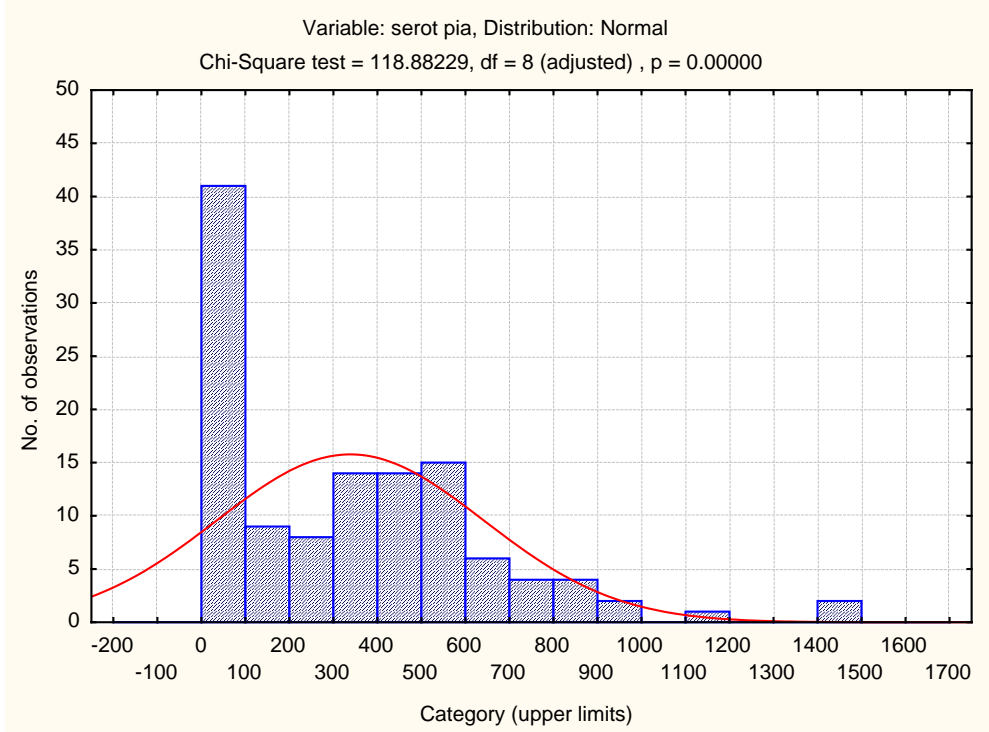

Figure 16. Platelet serotonin level and its distribution in the number of cases. Clearly, it's not normal.

Table 4. Biochemical parameters related to oxidative stress in CTR subjects and MD and BD patients. Data are normally distributed and expressed as mean $\pm \mathrm{SD}$.

\begin{tabular}{|c|c|c|c|c|}
\hline PARAMETERS & CTR & MD & BD & $p$ value (ANOVA) \\
\hline Lutein $(\mu \mathrm{mol} / \mathrm{l})$ & $0.34 \pm 0.14$ & $0.26 \pm 0.14^{\mathrm{a}}(-24 \%)$ & $0.27 \pm 0.13^{\mathrm{a}}(-21 \%)$ & 0.038 \\
\hline Lycopene $(\mu \mathrm{mol} / \mathrm{l})$ & $0.68 \pm 0.24$ & $0.57 \pm 0.22^{\mathrm{a}}(-16 \%)$ & $0.55 \pm 0.19^{\mathrm{a}}(-19 \%)$ & 0.022 \\
\hline Beta-carotene $(\mu \mathrm{mol} / \mathrm{l})$ & $0.73 \pm 0.49$ & $0.45 \pm 0.45^{\mathrm{a}}(-38 \%)$ & $0.43 \pm 0.29^{\mathrm{a}}(-41 \%)$ & 0.003 \\
\hline Retinol ( $\mu \mathrm{mol} / \mathrm{l})$ & $1.71 \pm 0.54$ & $1.54 \pm 0.49(-10 \%)$ & $1.57 \pm 0.58(-8 \%)$ & 0.445 \\
\hline Alpha-tocopherol $(\mu \mathrm{mol} / \mathrm{l})$ & $21.0 \pm 4.5$ & $20.1 \pm 5.6(-4 \%)$ & $20.7 \pm 4.9(-1 \%)$ & 0.791 \\
\hline oxLDL $(\mu \mathrm{g} / \mathrm{ml})$ & $1.31 \pm 0.54$ & $2.07 \pm 1.73^{\mathrm{a}}(+58 \%)$ & $1.52 \pm 1.14^{\mathrm{b}}(+16 \%)$ & 0.045 \\
\hline Homocysteine ( $\mu \mathrm{mol} / \mathrm{l})$ & $11.52 \pm 2.77$ & $13.94 \pm 5.29^{\mathrm{a}}(+21 \%)$ & $11.86 \pm 4.15^{\mathrm{b}}(+3 \%)$ & 0.047 \\
\hline
\end{tabular}

${ }^{\mathrm{a}}$ Significantly $(p<0.05)$ different from CTR; ' $\mathrm{b}$ Significantly $(p<0.05)$ different from MD.

Table 5. Biochemical parameters related to inflammation in CTR subjects and MD and BD patients. Data are not normally distributed and are expressed as mean (range).

\begin{tabular}{ccccc}
\hline PARAMETERS & CTR & MD & BD & $\begin{array}{c}p \text { value } \\
\text { (Kruskal-Wallis) }\end{array}$ \\
\hline IL-6 (pg/ml) & $43(8-80)$ & $44(5-101)$ & $56(8-126)$ & 0.403 \\
TNF-alpha (pg/ml) & $6.1(0.1-34.4)$ & $13.3(0.1-43.9)(+118 \%)$ & $8.9(0.1-46.0)(+46 \%)$ & 0.201 \\
LTB4 (pg/ml) & $221(115-377)$ & $265(84-522)(+20 \%)$ & $293(44-595)(+33 \%)$ & $284(5-958)(+65 \%)$ \\
PGE2 (pg/ml) & $172(43-376)$ & $332(20-1025)(+93 \%)$ & 0.241 & 0.333 \\
TXB2 (pg/ml) & $2405(710-4167)$ & $3095(18-13,389)(+29 \%)$ & $2994(49-14,644)(+24 \%)$ \\
CRP (mg/d) & $0.25(0.1-1.6)$ & $0.41(0.1-4.7)$ & $0.55(0.1-2.9)$ & 0.918 \\
ESR (mm/h) & $7(2-17)$ & $13(2-38)$ & 0.018 & 0.046 \\
\hline
\end{tabular}


detected in MD and BD patients as compared to CTR group; in particular, MD and BD groups presented high levels of TNF-alpha and PGE2. Differences were not significant probably due to the wide distribution range of the plasmatic levels of cytokines and eicosanoids. Significant differences were found among the three groups as regards ESR and CRP levels. Indeed, CRP and ESR strongly accumulated both in MD and BD patients as compared to CTR subjects.

\section{Discussion}

The results obtained in the present study have led to the achievement of an important scientific goal: a particular and specific composition of platelets FAs correlates significantly with the clinical state of depression and is therefore a fundamental support to the diagnosis of depression and, even more surprisingly, allows discriminating depressed subjects from bipolar ones. Consequently, different concentrations and relationships of platelet FAs are biological indicators of MD or BD. In detail, MD and BD patients showed to have a significant difference in PA, LA and AA platelet concentration, leading to relevant differences in membrane viscosity, as revealed by the Self Organising Map (SOM). Indeed, a negative B2 index (i.e. lower viscosity) was associated to $\mathrm{MD}$ and a positive B2 index (i.e. higher viscosity) to BD.

Both instruments (SOM and B2) should be used because some subjects have a very narrow range between negative and positive B2 index, even though, accurate psychiatric diagnosis, established with precision the nature of the disorder, i.e. negative B2 corresponding to MD and positive B2 to BD.

The power of the SOM, because it is impossible to manipulate it, is absolutely high and strength. The data entry in the SOM of the subjects who did not manifest an expressed pathology but divided between positive and negative indexes confirmed that they were not distinguishable from pathological cases and they were distributed over the SOM.

In particular, the index of membrane viscosity is stable over time in the same subject, and is independent from age, gender, dietetic behavior and therapy. The negative index is peculiar of the MD condition and the positive index is peculiar of $\mathrm{BD}$. The index does not change because of the high stability of the platelet fatty acids and because extensive clinical evaluations have only showed slight differences within the same sign without changing its positivity or negativity. Therefore, the membrane index, together with the SOM, can be proposed as a stable and peculiar marker of the pathological condition (MD and BD).

This result could open, in the immediate future of the field of psychiatry, the possibility of a new early diagnostic approach, considering that the affective syndromes are the most common psychiatric conditions and the second cause of disability in the world. In addition, the clinical characteristics, largely overlapping in MD disorder and in the depressive phase of $\mathrm{BD}$, the anamnestic difficulties and the many different ways of presentation of bipolar illness, are features that make even more complex the formulation of a specific early diagnosis, leading to the risk of administering inappropriate therapies that can adversely modify the evolution of the disorder, significantly reduce the quality of life of the patients and increase the risk of suicide [Tenth World Day for the Prevention of Suicide, Rome, 2012].

In fact, the use of antidepressants in BD is associated, with a poor efficacy and an increased risk of bias, to hypomanic or manic symptoms, to induction of rapid cycling, mixed states and chronicity of the disorder. The combination of an antidepressant with a mood stabilizer reduces but does not eliminate the risks associated with the use of antidepressants in bipolar patients.

A targeted diagnosis also means the reduction of side effects and a better quality of life of the subject. For the first time, the use of biological markers for the diagnosis of depression by a simple blood test means, in practical terms, to be really of help to the clinical diagnosis of this disease state that has been entrusted, so far, only to the clinic and the professionalism of psychiatrists.

The index of platelet membrane viscosity was also evaluated in apparently healthy volunteers, showing both positive and negative B2 values. The correspondence of the indices of CTR subjects to those of pathological patients involves some very important considerations. The deep diagnosis performed on CTR through the psychiatric tools, has documented, in many cases, the existence of a family history of mood disorders. It is possible that many people have a molecular similarity to that observed in pathological subjects but that do not express the disease, or that they have not yet expressed the disease, or that epigenetic phenomena must be considered critical to the rise of the pathology.

The similarity between platelet and neuron has been discussed (Cocchi, Tonello, De Lucia et al., 2009a; Cocchi, Tonello, De Lucia et al., 2009b; Cocchi, Sardi, Tonello et al., 2009) and explained by the hypothesis of the 
exchange of AA between platelets and neurons, regular in normal conditions; on the contrary, when AA is very high in platelets, as in the case of MD, the exchange terminates and AA increases in brain, increasing the fluidity of the membranes. Already in 1980 it has been shown a relationship between serotonin receptors and lipid membrane fluidity (Heron, Shinitzky, Hershkowitz et al., 1980; Lee, 1985): as the membrane become more viscous, the specific binding of serotonin increases steadily. Indeed, signal transduction, either through activation of adenylate cyclase by the ligand-receptor complex or by micro aggregation of ligand-receptor complexes, is associated with lateral movements of components of the membrane which are determined, at least partially, by lipid fluidity. In this context, we observed that within the control group, subjects with a positive platelet membrane viscosity index had higher levels of platelet serotonin than those with a negative index, thus confirming that a reduced cell membrane viscosity (as also evidenced in MD patients) reduces serotonin uptake.

In relation to oxidative stress, our study revealed a decrement of the antioxidant defense system in patients with MD and BD, in particular as regards carotenoid plasma levels. Carotenoids, considered a good indicator of fruit and vegetable intake, are efficient quenchers of singlet oxygen species and can directly scavenge free radicals. Low plasma concentrations of carotenoids have been recently associated with depressive symptoms and may predict the development of new depressive symptoms in older persons (Milaneschi, Bandinelli, Penninx et al., 2012).

Understanding the mechanism of this association may reveal potential targets for prevention and treatment. In this context, the therapeutic potential of antioxidant compounds as a co-adjuvant treatment to conventional antidepressants might be of clinical relevance (Scapagnini, Davinelli, Drago et al., 2012; Pandya, Howell, \& Pillai, 2012).

Indeed, even though antioxidant treatment for MD and BD is still in its infancy, current evidence suggests some beneficial value of various antioxidants, such as N-acetyl-cysteine and curcumin (Kulkarni, Bhutani, \& Bishnoi, 2008; Berk, Dean, Cotton et al., 2011).

A lowered antioxidant capacity is known to impair the protection against reactive oxygen species (ROS), which eventually cause damage to membrane lipids and lipoproteins. Accordingly, in MD patients we found a significant accumulation of oxidized LDL, which constitutes a major risk factor for genesis of atherosclerosis (Mitra, Goyal, \& Mehta, 2011). At the same time, MD subjects also presented high levels of homocysteine which is an independent risk factor for heart disease; indeed, homocysteine can induce oxidative stress promoting oxidant injury to vascular and blood cells (Dionisio, Jardín, Salido et al., 2010).

This evidence may further explain the higher incidence of coronary artery disease in MD disorder (Frasure-Smith \& Lespérance, 2006; Maes, Mihaylova, Kubera et al., 2010).

In depression, the induction of ROS pathways takes part in a more general inflammatory response characterized by an activation of cell-mediated immunity and increased levels of proinflammatory cytokines (McNamara \& Lotrich, 2012). Accordingly, MD patients showed high levels of TNF-alpha as compared to CTR subjects. BD patients also presented an accumulation of TNF-alpha; in fact, inflammation appears relevant to BD with altered cytokine levels during symptomatic (i.e., mania and depression) and asymptomatic intervals (Berk, Kapczinski, Andreazza et al., 2011). The same trend was observed for other markers of inflammation, namely eicosanoids, CRP and VES, demonstrating an elevated immune-inflammatory signaling in mood disorders (McNamara \& Lotrich, 2012).

This paper presents some aspects of complexity and difficulty of interpretation with respect to the traditional psychiatric evaluations. It is, therefore, necessary to clarify some of the problems that concern the methodological choices.

The results obtained showed that the platelet fatty acids composition is less variable than that of the plasma and red cell membrane, under nutritional influence. The index of membrane viscosity is stable in the same subject over time. A condition of greater or lesser clinical compensation corresponds to variations which are not substantial and don't show any transitions from a positive value to a negative or vice versa, and are not influenced by therapy. Further, Stahl et al., 1983, have concluded their work by commenting: "These platelet findings could not be explained by age, sex, or medication variables. The authors suggest that "the pharmacodynamics of platelet serotonin may be different in chronic schizophrenia than in bipolar major depressive disorder".

From the theoretical point of view it is plausible to think that platelets cannot undergo significant changes of the fatty acid composition unless of a significant dysregulation of the membrane functions and, therefore, induction of phenomena that can alter the delicate functions of the same platelets with undesirable consequences. 
Also, with regard to methodological approach, some considerations are possible with respect to the experimental choices made. In this regard it is appropriate a mathematical and methodological reasoning.

The search for "confounding variables" is a cornerstone of Evidence Based Medicine (EBM). This is correct because the EBM is based on standardized analytical methods, almost all, of statistical and probabilistic nature. Unlikely, our approach, uses the method of Artificial Neural Networks (Self Organizing Map-SOM), which is only partly statistical. It is therefore necessary to consider it in different way.

To build the SOM, a population of subjects with a generic diagnosis of Mood Disorders, without distinction within them between Major Depression and Bipolar Disorder, and a population of supposed healthy subjects was considered. Both populations were heterogeneous for sex and age. In addition, the subjects were treated with different drugs according to the classical psychiatric diagnosis. The Network has classified the subjects mapping (i.e. placing on the SOM map) pathologic subjects all close together and all healthy ones close together as well. The healthy subjects were far from the pathologic ones. The SOM placed them working "blindly", i.e. without knowing who was healthy or depressed, but she did so only on the basis of logic similarity of the numbers that were administered.

Since the three fatty acids were the only information administered to the SOM, in these three fatty acids there is something that is common within the pathological subjects and something that is common within the healthy population.

Suppose, with the logical approach known as "reduction ad absurdum", that the grouping criterion is not the presence of the disease, but any other variable, e.g. a confounding variable. Suppose, for example, that the confounding variable is the gender: this cannot be, because the SOM would have put females all close together and males all close together. It's obvious that it is not so, then gender is not a criterion that the SOM has taken into account. It is possible to see the same idea looking at the uniform distribution of the subjects within the map, from the gender point of view or looking at the absolute impossibility of identifying a "gender cluster".

We can also think about drugs: if the criterion were drugs, considering that the whole group of the depressed subjects was under very different medications, we should think that any type of drug used by pathologic subjects, although different, should have acted in exactly the same way on the balance of fatty acids investigated having mapped them all close, which is extremely unlikely, almost impossible.

It can be further evidenced by the fact that all pathological cases analyzed, before initiating therapy, have been mapped in the pathologic area, indeed, overlapping themselves to the pathologic subjects (supposed biased by drugs). This further exculpates the therapy from being a determining factor. The experimental choice, then, was made in order to identify the possibility of a marker that was very strong and able to classify the different psychiatric conditions. Evidently within the platelets is written something unmistakable.

In fact, using the same fatty acids but, by independent data from the first SOM, because collected in another region by different researchers and analyzed in different laboratory, with different tools, they have allowed the construction of a second SOM with the same logic. There may be the same "bias"?

\section{Conclusion}

This decade has clocked the review of the new DSM, the fifth in the series, the instrument considered the "bible" of psychiatry worldwide. The document, which is accomplished today, reveals firmly rooted in conservative psychiatric tradition ignoring the progress made by the biological research field. Of particular interest are the statements of NIMH (National Institute of Mental Health) by the voice of its director, who points out a strong critique to the DSM-V for not having considered the biological aspects of psychopathology. Clearly, the dichotomy between conservative and progressive psychiatry is not over despite the efforts of the scientific research in the field of psychiatry. The present work, shows, however, with the use of a unequivocal tool (the mathematical function that oversees the achievement of the SOM) that a biological aspect (platelets fatty acids), at least in the case of MD and BD, constitutes a determinant chance in the discrimination between the two psychopathologies. Why this possibility is written in platelets and which is the relationship that the SOM finds among the three FAs, to date, has not been yet known. Nevertheless, this finding certainly joins the chorus of the researches that recognize in mood disorders a clear genetic liability, which will be expressed in circumstances that, at the moment, we cannot identify and, perhaps, know in their fullness.

Recently some major events have allowed a movement of thought not only innovative, but of criticism, mainly at a high intellectual and scientific level, on the ideological implications of psychiatric diagnosis and of the increasing complexity of the nuances that classify the psychiatric disorder, rather than looking at a window 
that allows, through biological markers, a reliable diagnosis and appropriate care in the first diagnostic instance by limiting the diagnostic error unaware that psychiatric diagnosis has dragged on for years about the recognition of bipolar disorder from major depressive disorder where there is a diagnostic misinterpretation ranging from 40\% (Bowden, 2001) to 70\% [Tenth World Day for the Prevention of Suicide, Rome, 2012].

The complex experimentation carried out and described in this work moves between confirmations and innovative results. In the progression of the results, in fact, it demonstrates and confirms that inflammation and oxidative stress are related to mood disorders.

Recently (Khairova, Pawar, Salvadore et al., 2012) a significant decrease in the Superoxide/Catalase (SOD/ CAT) ratio was observed following lithium treatment, which was associated with decreased OxS by lowering hydrogen peroxide levels. This reduction in the SOD/CAT ratio may lead to lower OxS, indicated primarily by a decrease in the concentration of cell hydrogen peroxide. Overall, the present findings indicate a potential role for the antioxidant effects of lithium in healthy subjects, supporting its neuroprotective profile in bipolar disorder (BD) and, possibly, in neurodegenerative processes.

An element which must be considered concerns the fact that the experimental evidence, that led to the recognition of individuals with MD and BD, is due to a set of three FAs identified by the SOM, namely PA, LA, AA, corresponding to an evaluation obtained by a nonlinear mathematical function.

In short, we can conclude that the experimentation described in the work confirms that the artificial neural network (SOM) and the B2 index through the identification of three platelet fatty acids allow the classification of major depression and bipolar disorder, with the significant advantage of being able to address a correct therapeutic approach since the first diagnostic evaluation.

\section{Acknowledgements}

This study was supported by a grant of Regione Marche, Italy (special project). We are grateful to the staff of "Dipartimento di Salute Mentale" of Fano for assistance in blood sample collection. Most of all, thanks to all patients and healthy volunteers for their selfless donation of samples used in this study.

\section{Conflict of Interest}

The authors declare that they have no conflict of interest.

\section{References}

Aebischer, C. P., Schierle, J., \& Schuep, W. (1999). Simultaneous Determination of Retinol, Tocopherols, Carotene, Lycopene and Xanthophylls in Plasma by Means of Reversed-Phase High-Performance Liquid Chromatography. Methods in Enzymology, 299, 348-362. http://dx.doi.org/10.1016/S0076-6879(99)99035-3

Andreazza, A. C., Kauer-Sant’anna, M., Frey, B. N., Bond, D. J., Kapczinski, F., Young, L. T., \& Yatham, L. N. (2008). Oxidative Stress Markers in Bipolar Disorder: A Meta-Analysis. Journal of Affective Disorders, 111, 135-144. http://dx.doi.org/10.1016/j.jad.2008.04.013

Angst, J., Azorin, J. M., Bowden, C. L., Perugi, G., Vieta, E., Gamma, A., Young, A. H., \& BRIDGE Study Group (2011). Prevalence and Characteristics of Undiagnosed Bipolar Disorders in Patients with a Major Depressive Episode: The BRIDGE Study. JAMA Psychiatry, 68, 791-798. http://dx.doi.org/10.1001/archgenpsychiatry.2011.87

Bellivier, F., Yon, L., Luquiens, A., Azorin, J. M., Bertsch, J., \& Gerard, S. (2011). Suicidal Attempts in Bipolar Disorder: Results from an Observational Study (EMBLEM). Bipolar Disorders, 13, 377-386.

http://dx.doi.org/10.1111/j.1399-5618.2011.00926.x

Berk, M., Dean, O., Cotton, S. M., Gama, C. S., Kapczinski, F., Fernandes, B. S. et al. (2011). The Efficacy of N-Acetylcysteine as an Adjunctive Treatment in Bipolar Depression: An Open Label Trial. Journal of Affective Disorders, 135, 389-394. http://dx.doi.org/10.1016/j.jad.2011.06.005

Berk, M., Kapczinski, F., Andreazza, A. C., Dean, O. M., Giorlando, F., Maes, M., Yücel, M., Gama, C. S., Dodd, S., Dean, B., Magalhães, P. V. S., Amminger, P., McGorry, P., \& Malh, G. S. (2011). Pathways Underlying Neuroprogression in Bipolar Disorder: Focus on Inflammation, Oxidative Stress and Neurotrophic Factors. Neuroscience \& Biobehavioral Reviews, 35, 804-817. http://dx.doi.org/10.1016/j.neubiorev.2010.10.001

Bilici, M., Efe, H., Köroğlu, M. A., Uydu, H.A., Bekaroğlu, M., \& Değer, O. (2001). Antioxidative Enzyme Activities and Lipid Peroxidation in Major Depression: Alterations by Antidepressant Treatments. Journal of Affective Disorders, 64, 43-51. http://dx.doi.org/10.1016/S0165-0327(00)00199-3

Bowden, C. L. (2001). Strategies to Reduce Misdiagnosis of Bipolar Depression. Psychiatric Services, 52, 51-55. 
http://dx.doi.org/10.1176/appi.ps.52.1.51

Britt, S. G., Chiu, V. W., Redpath, G. T., \& VandenBerg, S. R. (1992). Elimination of Ascorbic Acid-Induced Membrane Lipid Peroxidation and Serotonin Receptor Loss by Trolox-C, a Water Soluble Analogue of Vitamin E. Journal of Receptor Research, 12, 181-200.

Camacho, A., \& Dimsdale, J. E. (2000). Platelets and Psychiatry: Lessons Learned from Old and New Studies. Psychosomatic Medicine, 62, 326-336. http://dx.doi.org/10.1097/00006842-200005000-00006

Cocchi, M., Tonello, L., Cappello, G., Tarozzi, G., Nabacino, L., Pastorini, E., Bucciarelli, S., Solazzo, L., De luca, M., Visci, G., \& Caramia, G. (2008). Membrane Platelet Fatty Acids: Biochemical Characterisation of the Ischemic Cardiovascular Disease, Characteristics of the Paediatric Age, through an Artificial Neural Network Interpretation. Medical and Surgical Pediatrics, 30, 25-30.

Cocchi, M., Gabrielli, F., Tonello, L., \& Pregnolato, M. (2010). The Interactome Hypothesis of Depression. NeuroQuantology, 8, 603-613.

Cocchi, M., Sardi, L., Tonello, L., \& Martelli, G. (2009). Do Mood Disorders Play a Role on Pig Welfare? Italian Journal of Animal Science, 8, 691-704.

Cocchi, M., \& Tonello, L. (2006). Biological, Biochemical and Mathematical Considerations about the Use of an Artificial Neural Network (ANN) for the Study of the Connection between Platelet Fatty Acids and Major Depression. Journal of Biological Research, 81, 82-87.

Cocchi, M., \& Tonello, L. (2007). Platelets, Fatty Acids, Depression and Cardiovascular Ischemic Pathology. Progress in Nutrition, 9, 94-104.

Cocchi, M., \& Tonello, L. (2010a). Bio Molecular Considerations in Major Depression and Ischemic Cardiovascular Disease. Central Nervous System Agents in Medicinal Chemistry, 10, 97-107. http://dx.doi.org/10.2174/187152410791196378

Cocchi, M., \& Tonello, L. (2010b). Running the Hypothesis of a Bio Molecular Approach to Psychiatric Disorder Characterization and Fatty Acids Therapeutical Choices. Annals of General Psychiatry, 9, S26. http://dx.doi.org/10.1186/1744-859X-9-S1-S26

Cocchi, M., Tonello, L., \& Cappello, G. (2006). Biochemical Markers in Major Depression as Interface between Neuronal Network and Artificial Neural Network (ANN). Journal of Biological Research, 81, 77-81.

Cocchi, M., Tonello, L., De Lucia, A., \& Amato, P. (2009a). Platelet and Brain Fatty Acids: A Model for the Classification of the Animals? Part 1. International Journal of Anthropology, 24, 69-76.

Cocchi, M., Tonello, L., De Lucia, A., \& Amato, P. (2009b). Platelet and Brain Fatty Acids: A Model for the Classification of the Animals? Part 2. Platelet and Brain Fatty Acid Transfer: Hypothesis on Arachidonic Acid and Its Relationship to Major Depression. International Journal of Anthropology, 24, 201-220.

Cocchi, M., Tonello, L., \& Gabrielli, F. (2012). Considerations on Blood Platelets: A Neuron’s Mirror for Mood Disorders? Open Journal of Blood Diseases, 2, 22-29. http://dx.doi.org/10.4236/ojbd.2012.22005

Cocchi, M., Tonello, L., \& Lercker, G. (2010). Fatty Acids, Membrane Viscosity, Serotonin and Ischemic Heart Disease. Lipids in Health and Disease, 9, 97. http://dx.doi.org/10.1186/1476-511X-9-97

Cocchi, M., Tonello, L., \& Rasenick, M. (2010). Human Depression: A New Approach in Quantitative Psychiatry. Annals of General Psychiatry, 9, 25. http://dx.doi.org/10.1186/1744-859X-9-25

Cocchi, M., Tonello, L., Tsaluchidu, S., \& Puri, B. K. (2008). The Use of Artificial Neural Networks to Study Fatty Acids in Neuropsychiatric Disorders. BMC Psychiatry, 8, S3. http://dx.doi.org/10.1186/1471-244X-8-S1-S3

Da Prada, M., Cesura, A. M., Launay, J. M., \& Richards, J. G. (1988). Platelets as a Model for Neurones? Experientia, 44, 115-126. http://dx.doi.org/10.1007/BF01952193

Dionisio, N., Jardín, I., Salido, G. M., \& Rosado, J. A. (2010). Homocysteine, Intracellular Signaling and Thrombotic Disorders. Current Medicinal Chemistry, 17, 3109-3119. http://dx.doi.org/10.2174/092986710791959783

Donati, R. J., Dwivedi, Y., Roberts, R. C., Conley, R. R., Pandey, G. N., \& Rasenick, M. M. (2008). Postmortem Brain Tissue of Depressed Suicides Reveals Increased Gs Localization in Lipid Raft Domains Where It Is Less Likely to Activate Adenylyl Cyclase. Journal of Neuroscience, 28, 3042-3050. http://dx.doi.org/10.1523/JNEUROSCI.5713-07.2008

Dowlati, Y., Herrmann, N., Swardfager, W., Liu, H., Sham, L., Reim, E. K., \& Lanctôt, K. L. (2010). A Meta-Analysis of Cytokines in Major Depression. Biological Psychiatry, 67, 446-457. http://dx.doi.org/10.1016/j.biopsych.2009.09.033

Engelberg, H. (1992). Low Serum Cholesterol and Suicide. Lancet, 339, 727-729. http://dx.doi.org/10.1016/0140-6736(92)90609-7

Evers, C. A., \& Starr, L. (2006). Biology: Concepts and Applications (6th ed.). Thomson.

Folch, J., Less, M., \& Sloane Stanley, G. H. (1957). A Simple Method for the Isolation and Purification of Total Lipids from Animal Tissue. Journal of Biological Chemistry, 226, 497-509. 
Fountoulakis, K. N. (2010). Pharmaceutical Treatment of Acute Bipolar Depression. F1000 Medicine Report 2. http://f1000.com/reports/m/2/47

Frasure-Smith, N., \& Lespérance, F. (2006). Depression and Coronary Artery Disease. Heart, 31, 64-68.

Goldstein, B. I., Kemp, D. E., Soczynska, J. K., \& McIntyre, R. S. (2009). Inflammation and the Phenomenology, Pathophysiology, Comorbidity, and Treatment of Bipolar Disorder: A Systematic Review of the Literature. Journal of Clinical Psychiatry, 70, 1078-1090. http://dx.doi.org/10.4088/JCP.08r04505

Heron, D. S., Shinitzky, M., Hershkowitz, M., \& Samuel, D. (1980). Lipid Fluidity Markedly Modulates the Binding of Serotonin to Mouse Brain Membranes. Proceedings of the National Academy of Sciences of the United States of America, 77, 7463-7467. http://dx.doi.org/10.1073/pnas.77.12.7463

Khairova, R., Pawar, R., Salvadore, G., Juruena, M. F., De Sousa, R. T., Soeiro-De-Souza, M. G., Salvador, M., Zarate, C. A., Gattaz, W. F., \& Machado-Vieira, R. (2012). Effects of Lithium on Oxidative Stress Parameters in Healthy Subjects. Molecular Medicine Reports, 5, 680-682. http://dx.doi.org/10.3892/mmr.2011.732

Kohonen, T. (2001). Self-Organizing Maps (3rd ed.). Berlin: Springer. http://dx.doi.org/10.1007/978-3-642-56927-2

Kulkarni, S. K., Bhutani, M. K., \& Bishnoi, M. (2008). Antidepressant Activity of Curcumin: Involvement of Serotonin and Dopamine System. Psychopharmacology, 201, 435-442. http://dx.doi.org/10.1007/s00213-008-1300-y

Lee, R. E. (1985). Membrane Engineering to Rejuvenate the Ageing Brain. Canadian Medical Association Journal, 132, 325-327.

Leonard, B. (2000). Clinical Implications of Mechanisms of Action of Antidepressants. Advances in Psychiatric Treatment, 6, 178-186. http://dx.doi.org/10.1192/apt.6.3.178

Maes, M., Galecki, P., Chang, Y. S., \& Berk, M. (2011). A Review on the Oxidative and Nitrosative Stress (O\&NS) Pathways in Major Depression and Their Possible Contribution to the (Neuro) Degenerative Processes in that Illness. Progress in Neuro-Psychopharmacology and Biological Psychiatry, 35, 676-692.

http://dx.doi.org/10.1016/j.pnpbp.2010.05.004

Maes, M., Mihaylova, I., Kubera, M., Uytterhoeven, M., Vrydags, N., \& Bosmans, E. (2010). Increased Plasma Peroxides and Serum Oxidized Low Density Lipoprotein Antibodies in Major Depression: Markers that Further Explain the Higher Incidence of Neurodegeneration and Coronary Artery Disease. Journal of Affective Disorders, 125, 287-294. http://dx.doi.org/10.1016/j.jad.2009.12.014

Mann, J. J. (2003). Neurobiology of Suicidal Behaviour. Nature Reviews Neuroscience, 4, 819-828. http://dx.doi.org/10.1038/nrn1220

Mann, J. J., \& Currier, D. M. (2010). Stress, Genetics and Epigenetic Effects on the Neurobiology of Suicidal Behavior and Depression. European Psychiatry, 25, 268-271. http://dx.doi.org/10.1016/j.eurpsy.2010.01.009

Marangos, P. J., Campbell, I. C., Schmechel, D. E., Murphy, D. L., \& Goodwin, F. K. (1980). Blood Platelets Contain a Neuron-Specific Enolase Subunit. Journal of Neurochemistry, 34, 1254-1258. http://dx.doi.org/10.1111/j.1471-4159.1980.tb09967.x

Mathews, D. C., Richards, E. M., Niciu, M. J., Ionescu, D. F., Rasimas, J. J., \& Zarate Jr., C. A. (2013). Neurobiological Aspects of Suicide and Suicide Attempts in Bipolar Disorder. Translational Neuroscience, 4, 203-216. http://dx.doi.org/10.2478/s13380-013-0120-7

Mazza, M., Di Nicola, M., Janiri, L., \& Bria, P. (2013). To Be or Not to Be a Bipolar Disorder Patient: Problems with Diagnosis. Journal of Nervous \& Mental Disease, 201, 435-437. http://dx.doi.org/10.1097/NMD.0b013e3182901de0

McNamara, R. K., \& Lotrich, F. E. (2012). Elevated Immune-Inflammatory Signaling in Mood Disorders: A New Therapeutic Target? Expert Review of Neurotherapeutics, 12, 1143-1161. http://dx.doi.org/10.1586/ern.12.98

Milaneschi, Y., Bandinelli, S., Penninx, B. W., Corsi, A. M., Lauretani, F., Vazzana, R. et al. (2012). The Relationship between Plasma Carotenoids and Depressive Symptoms in Older Persons. World Journal of Biological Psychiatry, 13, 588-598. http://dx.doi.org/10.3109/15622975.2011.597876

Mitra, S., Goyal, T., \& Mehta, J. L. (2011). Oxidized LDL, LOX-1 and Atherosclerosis. Cardiovascular Drugs and Therapy, 25, 419-429. http://dx.doi.org/10.1007/s10557-011-6341-5

Ng, F., Berk, M., Dean, O., \& Bush, A. I. (2008). Oxidative Stress in Psychiatric Disorders: Evidence Base and Therapeutic Implications. International Journal of Neuropsychopharmacology, 11, 851-876. http://dx.doi.org/10.1017/S1461145707008401

Nicholson, A. M., \& Ferreira, A. (2009). Increased Membrane Cholesterol Might Render Mature Hippocampal Neurons More Susceptible to Beta-Amyloid-Induced Calpain Activation and tau Toxicity. Journal of Neuroscience, 29, $4640-4651$. http://dx.doi.org/10.1523/JNEUROSCI.0862-09.2009

Ozcan, M. E., Gulec, M., Ozerol, E., Polat, R., \& Akyol, O. (2004). Antioxidant Enzyme Activities and Oxidative Stress in Affective Disorders. International Clinical Psychopharmacology, 19, 89-95. 
Pandey, G. N. (2011). Neurobiology of Adult and Teenage Suicide. Asian Journal of Psychiatry, 4, 2-13. http://dx.doi.org/10.1016/j.ajp.2011.01.008

Pandey, G. N., \& Dwivedi, Y. (2012). Peripheral Biomarkers for Suicide, In Y. Dwivedi (Ed.), The Neurobiological Basis of Suicide (pp. 407-424). Boca Raton, FL: CRC Press. http://dx.doi.org/10.1201/b12215-21

Pandya, C. D., Howell, K. R., \& Pillai, A. (2012). Antioxidants as Potential Therapeutics for Neuropsychiatric Disorders. Progress in Neuro-Psychopharmacology \& Biological Psychiatry, 46, 214-223.

Phillips, M. L., \& Kupfer, D. J. (2013). Bipolar Disorder Diagnosis: Challenges and Future Directions. Lancet, 381, 16631671. http://dx.doi.org/10.1016/S0140-6736(13)60989-7

Plein, H., \& Berk, M. (2001). The Platelet as a Peripheral Marker in Psychiatric Illness. Human Psychopharmacology, 16, 229-236. http://dx.doi.org/10.1002/hup.251

Pletscher, A., \& Laubscher, A. (1980). Blood Platelets as Models for Neurons: Uses and Limitations. Journal of Neural Transmission Supplementum, 16, 7-16.

Rawdin, B. J., Mellon, S. H., Dhabhar, F. S., Epel, E. S., Puterman, E., Su, Y., Burke, H. M., Reus, V. I., Rosser, R., Hamilton, S. P., Nelson, J. C., \& Wolkowitz, O. M. (2012). Dysregulated Relationship of Inflammation and Oxidative Stress in Major Depression. Brain, Behavior, and Immunity, 31, 143-152. http://dx.doi.org/10.1016/j.bbi.2012.11.011

Sachs, G. S. (1996). Bipolar Mood Disorder: Practical Strategies for Acute and Maintenance Phase Treatment. Journal of Clinical Psychopharmacology, 16, 32S-47S.

Scapagnini, G., Davinelli, S., Drago, F., De Lorenzo, A., \& Oriani, G. (2012). Antioxidants as Antidepressants: Fact or Fiction? CNS Drug, 26, 477-490. http://dx.doi.org/10.2165/11633190-000000000-00000

Smith, D. J., Griffiths, K. M., Hood, K., Craddock, N., \& Simpson, S. A. (2011). Unrecognised Bipolar Disorder in Primary Care Patients with Depression. British Journal of Psychiatry, 199, 49-56. http://dx.doi.org/10.1192/bjp.bp.110.083840

Stahl, S. M., Woo, D. J., Mefford, I. N., Berger, P. A., \& Ciaranello, R. D. (1983). Hyperserotonemia and Platelet Serotonin Uptake and Release in Schizophrenia and Affective Disorders. American Journal of Psychiatry, 140, 26-30.

Stanley, M., \& Stanley, B. (1989). Biochemical Studies in Suicide Victims: Current Findings and Future Implications. Suicide and Life-Threatening Behavior, 19, 30-42.

Stanley, M., Stanley, B., Traskman-Bendz, L., Mann, J. J., \& Meyendorff, E. (1986). Neurochemical Findings in Suicide Completers and Suicide Attempters. Suicide and Life-Threatening Behavior, 16, 286-300.

Steckert, A. V., Valvassori, S. S., Moretti, M., Dal-Pizzol, F., \& Quevedo, J. (2010). Role of Oxidative Stress in the Pathophysiology of Bipolar Disorder. Neurochemical Research, 35, 1295-1301.

http://dx.doi.org/10.1007/s11064-010-0195-2

Suppes, T., Datto, C., Minkwitz, M., Nordenhem, A., Walker, C., \& Darko, D. (2010). Effectiveness of the Extended Release Formulation of Quetiapine as Monotherapy for the Treatment of Acute Bipolar Depression. Journal of Affective Disorders, 121, 106-115. http://dx.doi.org/10.1016/j.jad.2009.10.007

Tonello, L., \& Cocchi, M. (2010). The Cell Membrane: Is It a Bridge from Psychiatry to Quantum Consciousness? NeuroQuantology, 8, 54-60.

Turecki, G. (2001). Suicidal Behavior: Is There a Genetic Predisposition? Bipolar Disorders, 3, 335-349. http://dx.doi.org/10.1034/j.1399-5618.2001.30608.x

Turecki, G., Ernst, C., Jollant, F., Labonte, B., \& Mechawar, N. (2012). The Neurodevelopmental Origins of Suicidal Behavior. Trends in Neurosciences, 35, 14-23. http://dx.doi.org/10.1016/j.tins.2011.11.008

Undurraga, J., Baldessarini, R. J., Valenti, M., Pacchiarotti, I., \& Vieta, E. (2012). Suicidal Risk Factors in Bipolar I and II Disorder Patients. Journal of Clinical Psychiatry, 73, 778-782. http://dx.doi.org/10.4088/JCP.11m07041

Yager, S., Forlenza, M. J., \& Miller, G. E. (2010). Depression and Oxidative Damage to Lipids. Psychoneuroendocrinology, 35, 1356-1362. http://dx.doi.org/10.1016/j.psyneuen.2010.03.010 